\title{
Birational geometry of moduli spaces of perverse coherent sheaves on blow-ups
}

\author{
Naoki Koseki ${ }^{1}$
}

Received: 26 July 2020 / Accepted: 19 April 2021 / Published online: 20 May 2021

(c) The Author(s) 2021

\begin{abstract}
In order to study the wall-crossing formula of Donaldson type invariants on the blown-up plane, Nakajima-Yoshioka constructed a sequence of blow-up/blow-down diagrams connecting the moduli space of torsion free framed sheaves on projective plane, and that on its blow-up. In this paper, we prove that Nakajima-Yoshioka's diagram realizes the minimal model program. Furthermore, we obtain a fully-faithful embedding between the derived categories of these moduli spaces.
\end{abstract}

\section{Contents}

1 Introduction . . . . . . . . . . . . . . . . . . . . . . . . . . . . 2380

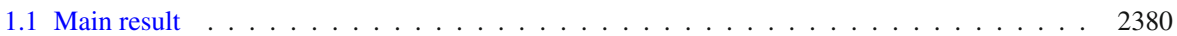

1.2 Strategy of the proof . . . . . . . . . . . . . . . . . . . . . . . 2381

1.3 Relation to existing works . . . . . . . . . . . . . . . . . . . . . . . 2381

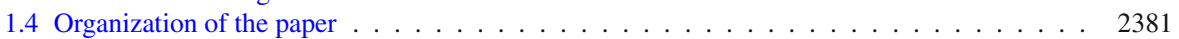

Notation and convention . . . . . . . . . . . . . . . . . . . . . . . . . . . 2382

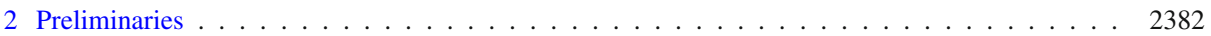

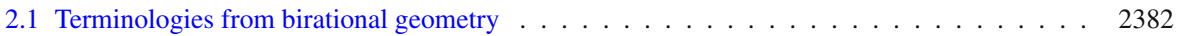

2.2 Fourier-Mukai functors . . . . . . . . . . . . . . . . . . . . . . . . 2383

2.3 Grassmannian flip . . . . . . . . . . . . . . . . . . . . . . . . . . . . . . 2384

2.4 Bott type vanishing . . . . . . . . . . . . . . . . . . . . . . . . . . . 2386

3 Framed sheaves on the blow-up . . . . . . . . . . . . . . . . . . . . . . . . . . . . 2387

3.1 ADHM description and wall crossing . . . . . . . . . . . . . . . . . . . . . 2387

3.2 Brill-Noether loci . . . . . . . . . . . . . . . . . . . . . . . . . . . . . . . . . . . . . . . 2389

4 Birational geometry of moduli spaces . . . . . . . . . . . . . . . . . . . . 2390

4.1 Birational geometry of moduli spaces _ . . . . . . . . . . . . . . . . . . 2391

4.2 Fully faithful embedding between derived categories . . . . . . . . . . . . . . . 2396

4.3 Projective case . . . . . . . . . . . . . . . . . . . . . . . . . . . . . . . . . 2397

5 Examples . . . . . . . . . . . . . . . . . . . . . . . . . . . 2398

5.1 Simplest example . . . . . . . . . . . . . . . . . . . . . . . . . . . 2398

5.2 Hilbert scheme of points . . . . . . . . . . . . . . . . . . . . . . . . . 2400

References . . . . . . . . . . . . . . . . . . . . . . . . . 2403

$\bowtie$ Naoki Koseki

nkoseki@ed.ac.uk

1 School of Mathematics and Maxwell Institute, University of Edinburgh, James Clerk Maxwell Building, Peter Guthrie Tait Road, Edinburgh EH9 3FD, United Kingdom 


\section{Introduction}

\subsection{Main result}

The wall crossing formulas of Donaldson-type invariants have been investigated in various papers. For example, the behavior of Donaldson invariants of the moduli spaces of rank two stable sheaves on rational surfaces under the variations of polarizations are studied by Ellingsrud and Göttsche [10], Friedman and Qin [11]. As another example, Nakajima and Yoshioka [21-23] studied the difference of invariants of framed sheaves on the projective plane $\mathbb{P}^{2}$ and that on its blow-up $\hat{\mathbb{P}}^{2}$ at a point. To do so, they constructed a sequence of diagrams

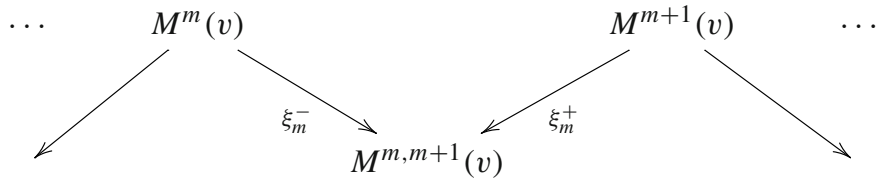

connecting the moduli space on $\mathbb{P}^{2}$ and that on the blow-up $\hat{\mathbb{P}}^{2}$. The intermediate models $M^{m}(v)$ also have modular interpretations; they are the moduli spaces of $m$-stable sheaves (see Definition 3.1). Similarly, the space $M^{m, m+1}(v)$ parametrizes $m$-stable and $(m+1)$-stable sheaves with various Chern characters.

In these examples, the moduli spaces appearing in wall crossing diagrams are smooth and birational to each other. In fact, in the case studied in $[10,11]$, the moduli spaces are connected by standard flips. In the case of [21-23], their geometry is more complicated. Indeed, Nakajima-Yoshioka proved that the contracted loci of the morphisms $\xi_{m}^{ \pm}$have stratifications (called Brill-Noether stratifications) such that each stratum has the structure of a Grassmannian bundle.

The aim of this paper is the further study of birational geometric properties of the diagram (1.1). In particular, we show that it is an instance of the minimal model program.

Theorem 1.1 (Theorem 4.7) The diagram (1.1) realizes a minimal model program for the moduli space of framed torsion free sheaves on the blow-up $\hat{\mathbb{P}}^{2}$. The program ends with the minimal model, the moduli space of framed torsion free sheaves on $\mathbb{P}^{2}$, which is a hyperKähler manifold.

We will also verify Bondal-Orlov [5], Kawamata's [15] D/K equivalence conjecture for these moduli spaces:

Theorem 1.2 (Theorem 4.9) For each integer $m \in \mathbb{Z}_{\geq 0}$, we have a fully faithful embedding

$$
D^{b}\left(M^{m}(v)\right) \hookrightarrow D^{b}\left(M^{m+1}(v)\right)
$$

between the derived categories. In particular, we have an embedding

$$
D^{b}\left(M_{\mathbb{P}^{2}}\right) \hookrightarrow D^{b}\left(M_{\hat{\mathbb{P}}^{2}}\right),
$$

where $M_{\mathbb{P}^{2}}, M_{\hat{\mathbb{P}}^{2}}$ denote the moduli spaces of torsion free framed sheaves on $\mathbb{P}^{2}, \hat{\mathbb{P}}^{2}$, respectively.

So we get an interesting relationship among wall-crossing formulas for Donaldson type invariants, birational geometry, and derived categories.

We can also consider the moduli space of Gieseker stable sheaves on a smooth projective surface and that on its blow-up (see Theorem 4.10 for the precise statement): 
Theorem 1.3 Let $S$ be a smooth projective surface, $\hat{S}$ the blow-up of $S$ at a point. Under certain numerical conditions, the MMP for the moduli space $M_{\hat{S}}$ of Gieseker stable sheaves on $\hat{S}$ is reduced to MMP for the moduli $M_{S}$ on S. Furthermore, there exists a fully faithful embedding

$$
D^{b}\left(M_{S}\right) \hookrightarrow D^{b}\left(M_{\hat{S}}\right)
$$

between their derived categories.

For instance, we can apply the above theorem when $S$ is a del Pezzo surface.

\subsection{Strategy of the proof}

To prove our main result Theorem 1.1, we will compute the normal bundles of the fibers explicitly, following the idea from [10,11]. Although the geometry of the diagram (1.1) is more complicated compared to the one considered in [10,11], it turns out their method still works in our setting. Actually, we are able to describe the normal bundle of each BrillNoether stratum explicitly. Then we will see that the diagram realizes the MMP when we decrease the stability parameter $m \in \mathbb{Z}_{\geq 0}$.

Furthermore, the normal bundle computation enables us to reduce the construction of the fully faithful embedding between derived categories to the formal local case; the latter is already handled in the paper [8] and hence we can prove Theorem 4.9.

\subsection{Relation to existing works}

In [17], the author studied birational geometry of the Hilbert scheme of two points on blowups. The main result of the present paper is an extension to the completely general setting.

There are several works investigating birational geometry and derived categories of moduli spaces. For a standard flip between moduli spaces obtained in [10,11], Ballard [4] constructed a semi-orthogonal decomposition (SOD) of their derived categories.

Recently, Toda [24,25] introduced the notion of d-critical birational geometry, which is a certain virtual analogue of usual birational geometry. It is shown that if two smooth varieties are connected by a simple d-critical flip, then we have an SOD of their derived categories. See $[18,25]$ for interesting examples of d-critical flips.

The SODs obtained in the papers [4], [25] can be considered as categorifications of wall crossing formulas for Donaldson type invariants, Donaldson-Thomas type invariants, respectively. It would be interesting to describe the semi-orthogonal complements of the embedding in our Theorem 4.9, which would give a categorification of Nakajima-Yoshioka's wall crossing formula.

\subsection{Organization of the paper}

The paper is organized as follows. In Sect. 2, we collect some terminology and useful lemmas from birational geometry and derived categories. In Sect. 3, we recall the result of NakajimaYoshioka. In Sect. 4, we prove our main results. In Sect. 5, we give some explicit examples. 


\section{Notation and convention}

In this paper, we always work over the complex number field $\mathbb{C}$.

- For a variety $X$, we denote by $D^{b}(X):=D^{b}(\operatorname{Coh}(X))$ the bounded derived category of coherent sheaves on $X$.

- For a proper morphism $f: M \rightarrow N$ between varieties and objects $E, F \in D^{b}(M)$, we denote by $\mathcal{E} x t_{f}^{q}(E, F)$ the $q$-th derived functor of $\mathcal{H o m}_{f}(E, F):=f_{*} \mathcal{H o m}(E, F)$.

- For coherent sheaves $E, F$ on a variety, we define $\operatorname{hom}(E, F):=\operatorname{dim} \operatorname{Hom}(E, F)$ and $\operatorname{ext}^{i}(E, F):=\operatorname{dim} \operatorname{Ext}^{i}(E, F)$.

- For a vector bundle $\mathcal{V}$ on a variety and an integer $i>0$, we denote by $\operatorname{Gr}(i, \mathcal{V})$ the Grassmann bundle of $i$-dimensional subbundles of $\mathcal{V}$.

\section{Preliminaries}

\subsection{Terminologies from birational geometry}

In this subsection, we recall some notions from birational geometry. The standard reference for this subsection is [16].

Definition 2.1 Let $\phi: X \rightarrow Z$ be a projective morphism between normal quasi-projective varieties. We say that $\phi$ is a $K$-positive (resp. $K$-negative) contraction if the following conditions hold:

(1) $\phi_{*} \mathcal{O}_{X} \cong \mathcal{O}_{Z}$,

(2) the canonical divisor $K_{X}$ (resp. the anti-canonical divisor $-K_{X}$ ) is $\phi$-ample.

Definition 2.2 Let $\phi: X \rightarrow Z$ be a $K$-negative contraction.

(1) $\phi$ is called a divisorial (resp. flipping) contraction if it is birational and the $\phi$-exceptional locus has codimension one (resp. at least two).

(2) $\phi$ is called a Mori fiber space if we have $\operatorname{dim} X>\operatorname{dim} Z$.

(3) Assume that $\phi$ is a flipping contraction. Then a flip of $\phi$ is a $K$-positive birational contraction $\phi^{+}: X^{+} \rightarrow Z$. We also call the birational map $X \rightarrow X^{+}$a flip.

Definition 2.3 Let $X$ be a quasi-projective variety with at worst terminal singularities. A minimal model program of $X$ is a sequence of birational maps

$$
X=X_{0} \rightarrow X_{1} \rightarrow \cdots--\rightarrow X_{N},
$$

such that

(1) each birational map $X_{i} \rightarrow X_{i+1}$ is either a divisorial contraction or a flip,

(2) the variety $X_{N}$ is either a minimal model (i.e. $K_{X_{N}}$ is nef) or has a structure of a Mori fiber space.

We do not give the definition of a terminal singularity, as we only consider smooth varieties in this paper. For the precise definition, see [16, Definition 2.34].

The following lemma is useful for our purpose:

Lemma 2.4 [1, Proposition 3.7] Let $X$ be a smooth variety, $\phi: X \rightarrow Z$ a K-negative contraction, and $F \subset X$ a smooth $\phi$-fiber. Assume that the following conditions hold: 
- the conormal bundle $\mathcal{N}^{\vee}:=\mathcal{N}_{F / X}^{\vee}$ is nef,

- $H^{1}\left(F, \mathcal{T}_{F} \otimes \operatorname{Sym}^{i}\left(\mathcal{N}^{\vee}\right)\right)=H^{1}\left(F, \mathcal{N} \otimes \operatorname{Sym}^{i}\left(\mathcal{N}^{\vee}\right)\right)=0$ for $i \geq 1$.

Then the formal neighborhood of $F$ in $X$ is isomorphic to that of $F$ in the total space of $\mathcal{N}$, embedded as the zero section.

\subsection{Fourier-Mukai functors}

In this subsection, we recall the definition and basic properties of Fourier-Mukai functors. The standard reference is [13].

Definition 2.5 Let $X, Y$ be smooth quasi-projective varieties, and $\mathcal{P} \in D^{b}(X \times Y)$ be an object whose support is proper over $Y$. The Fourier-Mukai $(F M)$ functor with kernel $\mathcal{P}$ is the functor $\Phi^{\mathcal{P}}: D^{b}(X) \rightarrow D^{b}(Y)$ defined by

$$
D^{b}(X) \ni E \mapsto \Phi^{\mathcal{P}}(E):=\mathbf{R} p_{Y *}\left(p_{X}^{*} E \otimes^{\mathbf{L}} \mathcal{P}\right) \in D^{b}(Y),
$$

where $p_{X}: X \times Y \rightarrow X, p_{Y}: X \times Y \rightarrow Y$ denote the projections.

Note that, in the above definition, we assume the object $\mathcal{P}$ has proper support over $Y$ to ensure the associated FM functor $\Phi^{\mathcal{P}}$ preserves bounded complexes.

Let us give a trivial example:

Example 2.6 Let $\mathcal{O}_{\Delta_{X}}$ be the structure sheaf of the diagonal $\Delta_{X} \subset X \times X$. Then the associated FM functor $\Phi^{\mathcal{O}_{\Delta_{X}}}$ coincides with the identity functor $\operatorname{id}_{D^{b}(X)}$.

Let $\Phi=\Phi^{\mathcal{P}}: D^{b}(X) \rightarrow D^{b}(Y)$ be a FM functor with kernel $\mathcal{P}$. By [13, Proposition 5.9], the right adjoint functor $\Phi^{\mathbf{R}}: D^{b}(Y) \rightarrow D^{b}(X)$ is given by the FM functor with kernel

$$
\mathcal{P}_{\mathbf{R}}:=\mathcal{P}^{\vee} \otimes p_{X}^{*} \omega_{X}[\operatorname{dim} X] .
$$

Similarly, the composition of two FM functors is again a FM functor: let $\Phi^{\mathcal{Q}}: D^{b}(Y) \rightarrow$ $D^{b}(Z)$ be another FM functor. Then the composition $\Phi^{\mathcal{Q}} \circ \Phi^{\mathcal{P}}: D^{b}(X) \rightarrow D^{b}(Z)$ is a FM functor with kernel

$$
\mathcal{P} * \mathcal{Q}:=\mathbf{R} p_{X Z *}\left(p_{X Y}^{*} \mathcal{P} \otimes{ }^{\mathbf{L}} p_{Y Z}^{*} \mathcal{Q}\right) \in D^{b}(X \times Z),
$$

where $p_{X Y}: X \times Y \times Z \rightarrow X \times Y, p_{Y Z}: X \times Y \times Z \rightarrow Y \times Z, p_{X Z}: X \times Y \times Z \rightarrow X \times Z$ denote the projections, see [13, Proposition 5.10].

Now let us consider the adjoint map

$$
\Phi^{\mathcal{O}_{\Delta_{X}}}=\operatorname{id}_{D^{b}(X)} \rightarrow \Phi^{\mathcal{P}_{\mathbf{R}}} \circ \Phi^{\mathcal{P}}=\Phi^{\mathcal{P}_{R} * \mathcal{P}} .
$$

The following result tells us that it lifts to the morphism between FM kernels:

Proposition 2.7 [2, Theorem 3.2, Proposition 3.6] Let $\Phi=\Phi^{\mathcal{P}}: D^{b}(X) \rightarrow D^{b}(Y)$ be a $F M$ functor. Then there exists a morphism

$$
\mathcal{O}_{\Delta_{X}} \rightarrow \mathcal{P}_{\mathbf{R}} * \mathcal{P}
$$

in $D^{b}(X \times X)$, which induces the adjoint map (2.1).

We will use the following lemma in the proof of the main result: 
Lemma 2.8 Let $X, Y$ be smooth quasi-projective varieties, $X \rightarrow U, Y \rightarrow U$ projective morphisms to a variety $U$. Take an object $\mathcal{P} \in D^{b}(X \times Y)$ supported on the fiber product $X \times_{U} Y$. Denote by $\mathcal{Q} \in D^{b}(X \times X)$ the cone of the morphism $\mathcal{O}_{\Delta_{X}} \rightarrow \mathcal{P}_{\mathbf{R}} * \mathcal{P}$ in (2.2). The following statements hold:

(1) The FM functor $\Phi=\Phi^{\mathcal{P}}$ is fully faithful if and only if $\mathcal{Q}=0$.

(2) For a point $p \in U$, denote by $\hat{U}_{p}$ the completion of $U$ at $p$. Denote by $\hat{X}_{p}$ the base change of $X \rightarrow U$ along $\hat{U}_{p} \rightarrow U$. Then $\mathcal{Q}=0$ if and only if $\mathcal{Q} \otimes_{\mathcal{O}_{X \times X}} \mathcal{O}_{\hat{X}_{p} \times \hat{X}_{p}}=0$ for every $p \in U$.

In particular, the FM functor $\Phi^{\mathcal{P}}$ is fully faithful if and only if the FM functors $\Phi^{\mathcal{P}} \hat{p}$ are fully faithful for all $p \in U$, where we put $\mathcal{P}_{p}^{\wedge}:=\mathcal{P} \otimes_{\mathcal{O}_{X \times Y}} \mathcal{O}_{\hat{X}_{p} \times \hat{Y}_{p}}$.

Proof (1) First observe that the functor $\Phi$ is fully faithful if and only if the adjoint map (2.1) is an isomorphism. Let us take an object $E \in D^{b}(X)$. By applying $\Phi^{(-)}(E)$ to the exact triangle

$$
\mathcal{O}_{\Delta_{X}} \rightarrow \mathcal{P}_{\mathbf{R}} * \mathcal{P} \rightarrow \mathcal{Q},
$$

in $D^{b}(X \times X)$, we obtain an exact triangle

$$
E \rightarrow \Phi^{\mathbf{R}} \circ \Phi(E) \rightarrow \Phi^{\mathcal{Q}}(E)
$$

in $D^{b}(X)$. Hence if $\mathcal{Q}=0$, we have an isomorphism $E \stackrel{\cong}{\rightarrow} \Phi^{\mathbf{R}} \circ \Phi(E)$ for all $E \in D^{b}(X)$, i.e., $\Phi$ is fully faithful.

For the converse, assume that $\mathcal{Q} \neq 0$. Then there exists a point $x \in X$ such that $\mathcal{Q}_{x}:=$ $\left.\mathcal{Q}\right|_{\{x\} \times X} \neq 0$. In particular, we have $\Phi^{\mathcal{Q}}\left(\mathcal{O}_{x}\right)=\mathcal{Q}_{x} \neq 0$ and hence $\mathcal{O}_{x} \neq \Phi^{\mathbf{R}} \circ \Phi\left(\mathcal{O}_{x}\right)$ by the exact triangle (2.3), i.e., the functor $\Phi$ is not fully faithful.

(2) The second assertion follows from the fact that the completion $\hat{U}_{p} \rightarrow U$ is faithfully flat.

\subsection{Grassmannian flip}

Here we recall about geometry and the derived categories of Grassmannian flips, which play important roles in this paper. We refer [3, Chapter II] for the details (see also [8, Section 1]). Let $W^{ \pm}$be vector spaces. Take a positive integer $i \leq \min \left\{\operatorname{dim} W^{ \pm}\right\}$.

Then the determinantal variety is defined to be

$$
Z=Z_{i}:=\left\{a \in \operatorname{Hom}\left(\left(W^{-}\right)^{\vee}, W^{+}\right): \operatorname{rk}(a) \leq i\right\},
$$

and its Springer resolution is defined as

$$
Y^{+}=Y_{i}^{+}:=\left\{(a, V) \in \operatorname{Hom}\left(\left(W^{-}\right)^{\vee}, W^{+}\right) \times \operatorname{Gr}\left(i, W^{+}\right): \operatorname{Image}(a) \subset V\right\} .
$$

We have the following projections:

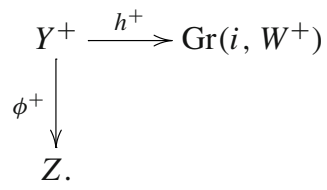

The fiber of $h^{+}: Y^{+} \rightarrow \operatorname{Gr}\left(i, W^{+}\right)$over a point $V \in \operatorname{Gr}\left(i, W^{+}\right)$is

$$
\operatorname{Hom}\left(\left(W^{-}\right)^{\vee}, V\right) \cong V \otimes W^{-} \text {. }
$$


Hence we have an isomorphism

$$
Y^{+} \cong \operatorname{Tot}_{\mathrm{Gr}\left(i, W^{+}\right)}\left(S^{+} \otimes W^{-}\right),
$$

where $S^{+}$denotes the universal subbundle on $\operatorname{Gr}\left(i, W^{+}\right)$. This shows that the variety $Y^{+}$is smooth of dimension $i\left(\operatorname{dim} W^{+}+\operatorname{dim} W^{-}-i\right)$. Moreover, the other projection $\phi^{+}: Y^{+} \rightarrow Z$ is isomorphism over the open locus

$$
Z^{o}:=\{a \in Z: \operatorname{rk}(a)=i\},
$$

therefore $Y^{+} \rightarrow Z$ is actually a resolution of singularities.

On the other hand, we have canonical isomorphisms

$$
\operatorname{Hom}\left(\left(W^{+}\right)^{\vee}, W^{-}\right) \cong W^{-} \otimes W^{+} \cong \operatorname{Hom}\left(\left(W^{-}\right)^{\vee}, W^{+}\right),
$$

hence by replacing the roles of $W^{ \pm}$in the above construction, we get a second resolution of singularities of the variety $Z$ :

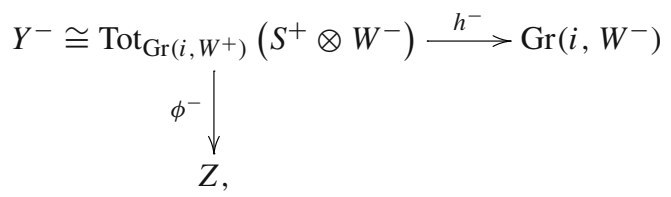

where $S^{-}$denotes the universal subbundle of $\operatorname{Gr}\left(i, W^{-}\right)$.

Hence we obtain the diagram

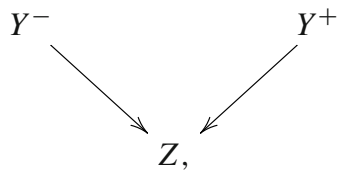

which we call the Grassmannian flip. The following lemma justifies this notion:

Lemma 2.9 Assume that $\operatorname{dim} W^{+}>\operatorname{dim} W^{-}$. Then the following statements hold:

(1) The canonical bundles of $Y^{ \pm}$are given as

$$
\omega_{Y^{ \pm}}=h^{ \pm *}\left(\operatorname{det}\left(S^{ \pm}\right)^{\otimes \pm\left(\operatorname{dim} W^{+}-\operatorname{dim} W^{-}\right)}\right) .
$$

In particular, the morphism $\phi^{+}$(resp. $\phi^{-}$) is a $K$-negative (resp. $K$-positive) contraction.

(2) When $i=\operatorname{dim} W^{-}$, the morphism $\phi^{+} i$ s a divisorial contraction and the morphism $\phi^{-}$ is an isomorphism.

(3) When $i<\operatorname{dim} W^{-}$, the birational map $Y^{+}{ }_{-\rightarrow} \rightarrow Y^{-}$is a flip.

Proof (1) We have the following exact sequence

$$
0 \rightarrow h^{ \pm *}\left(S^{ \pm} \otimes W^{\mp}\right) \rightarrow \mathcal{T}_{Y^{ \pm}} \rightarrow h^{ \pm *} \mathcal{T}_{\mathrm{Gr}\left(i, W^{ \pm}\right)} \rightarrow 0,
$$

and the tangent bundle of the Grassmannian variety is given by $\mathcal{T}_{\operatorname{Gr}\left(i, W^{ \pm}\right)} \cong S^{ \pm *} \otimes Q^{ \pm}$, where $Q^{ \pm}$denotes the universal quotient bundle of $\operatorname{Gr}\left(i, W^{ \pm}\right)$. Hence we obtain

$$
\begin{aligned}
\omega_{Y^{ \pm}} & \cong h^{ \pm *}\left(\operatorname{det}\left(S^{ \pm}\right)^{\otimes-\operatorname{dim} W^{\mp}} \otimes\left(\operatorname{det}\left(S^{ \pm}\right)^{\otimes\left(\operatorname{dim} W^{ \pm}-i\right)} \otimes \operatorname{det}\left(Q^{ \pm *}\right)^{\otimes i}\right)\right) \\
& \cong h^{ \pm *}\left(\operatorname{det}\left(S^{ \pm}\right)^{\otimes\left(\operatorname{dim} W^{ \pm}-\operatorname{dim} W^{\mp}\right)} \otimes\left(\operatorname{det}\left(S^{ \pm}\right) \otimes \operatorname{det}\left(Q^{ \pm}\right)\right)^{\otimes-i}\right) .
\end{aligned}
$$


Moreover, the tautological sequence

$$
0 \rightarrow S^{ \pm} \rightarrow W^{ \pm} \otimes \mathcal{O}_{\mathrm{Gr}\left(i, W^{ \pm}\right)} \rightarrow Q^{ \pm} \rightarrow 0
$$

shows that

$$
\operatorname{det}\left(S^{ \pm}\right) \otimes \operatorname{det}\left(Q^{ \pm}\right) \cong \mathcal{O}_{\mathrm{Gr}\left(i, W^{ \pm}\right)}
$$

Now Eq. (2.4) becomes

$$
\omega_{Y^{ \pm}} \cong h^{ \pm *}\left(\operatorname{det}\left(S^{ \pm}\right)^{\otimes\left(\operatorname{dim} W^{ \pm}-\operatorname{dim} W^{\mp}\right)}\right)
$$

as required.

To prove (2) and (3), we need to determine the dimensions of the $\phi^{ \pm}$-exceptional loci. First note that we have a sequence of closed immersions

$$
\{0\}=Z_{0} \subset Z_{1} \subset \cdots Z_{i-1} \subset Z_{i}=Z,
$$

and we have $\operatorname{dim}\left(\phi^{ \pm}\right)^{-1}\left(Z_{j}\right) \leq \operatorname{dim}\left(\phi^{ \pm}\right)^{-1}\left(Z_{i-1}\right)$ for all $j \leq i-1$.

Let us take a point $a \in Z_{i-1} \backslash Z_{i-2}$. By definition, the subspace $V_{a}:=\operatorname{Image}(a) \subset W^{ \pm}$ has dimension $i-1$. The fibers $Y_{a}^{ \pm}:=\left(\phi^{ \pm}\right)^{-1}(a)$ are

$$
Y_{a}^{ \pm} \cong\left\{V \in \operatorname{Gr}\left(i, W^{ \pm}\right): V_{a} \subset V\right\} \cong \operatorname{Gr}\left(1, W^{ \pm} / V_{a}\right) .
$$

Hence we have

$$
\begin{aligned}
\operatorname{dim} \operatorname{Exc}\left(\phi^{ \pm}\right) & =\operatorname{dim} Z_{i-1}+\operatorname{dim} \operatorname{Gr}\left(1, W^{ \pm} / V_{a}\right) \\
& =\operatorname{dim} Y_{i-1}^{ \pm}+\operatorname{dim} \operatorname{Gr}\left(1, W^{ \pm} / V_{a}\right) \\
& =(i-1)\left(\operatorname{dim} W^{ \pm}+\operatorname{dim} W^{\mp}-i+1\right)+\left(\operatorname{dim} W^{ \pm}-(i-1)-1\right) \\
& =i\left(\operatorname{dim} W^{ \pm}+\operatorname{dim} W^{\mp}-i\right)+i-\operatorname{dim} W^{\mp}-1 \\
& =\operatorname{dim} Y^{ \pm}-\left(\operatorname{dim} W^{\mp}+1-i\right),
\end{aligned}
$$

and the assertions (2) and (3) hold.

For the derived categories of $Y^{ \pm}$, we have the following result:

Theorem $2.10\left[8\right.$, Theorem D] Assume that $\operatorname{dim} W^{+}>\operatorname{dim} W^{-}$. Then the FM functor

$$
\Phi^{\mathcal{O}_{W}}: D^{b}\left(Y^{-}\right) \hookrightarrow D^{b}\left(Y^{+}\right)
$$

is fully faithful, where we put $W:=Y^{-} \times_{Z} Y^{+}$.

Remark 2.11 When $\operatorname{dim} W^{-}=\operatorname{dim} W^{+}$, the canonical bundles of $Y^{ \pm}$are trivial, and we call the birational map $Y^{+} \rightarrow Y^{-}$the Grassmannian flop. In this case, the FM functor $\Phi^{\mathcal{O}_{W}}$ is an equivalence, which is treated also in [9].

\subsection{Bott type vanishing}

For later use, we prove some vanishing results on cohomology groups of certain vector bundles on the Grassmannian varieties. Let $W$ be a vector space of dimension $n$, and $i \in \mathbb{Z}_{>0}$ be a positive integer with $i<n$. We consider the Grassmannian variety $G=\operatorname{Gr}(i, W)$. Denote by $S, Q$ the universal sub and quotient bundles, respectively. 
An element $\beta=\left(\beta_{1}, \ldots, \beta_{s}\right) \in \mathbb{Z}^{\oplus s}$ is called a weight if it satisfies $\beta_{1} \geq \beta_{2} \geq \cdots \geq$ $\beta_{s-1} \geq \beta_{s}$. For a given pair $\alpha=(\beta, \gamma)$ of weights $\beta \in \mathbb{Z}^{\oplus i}$ and $\gamma \in \mathbb{Z}^{\oplus n-i}$, we define the vector bundle $V(\alpha)$ as

$$
V(\alpha):=K_{\beta}\left(S^{\vee}\right) \oplus K_{\gamma}\left(Q^{\vee}\right),
$$

where $K_{\beta}(-), K_{\gamma}(-)$ denote the Weyl functors (cf. [26, Section 2.1]).

Example 2.12 (1) For $\alpha=(k, 0, \ldots, 0) \in \mathbb{Z}^{\oplus n}$ with $k \geq 0$, we have $V(\alpha)=\operatorname{Sym}^{k}\left(S^{\vee}\right)$.

(2) For $\alpha=(0, \ldots, 0,-1) \in \mathbb{Z}^{\oplus n}$, we have $V(\alpha)=Q$.

We will use the following version of Bott vanishing:

Theorem 2.13 Assume that an element $\alpha=\left(\beta_{1}, \ldots, \beta_{i}, \gamma_{i+1}, \ldots, \gamma_{n}\right) \in \mathbb{Z}^{\oplus n}$ satisfies $\beta_{1} \geq$ $\ldots \beta_{i} \geq \gamma_{i+1} \geq \cdots \geq \gamma_{n}$. Then we have the vanishing

$$
H^{j}(G, V(\alpha))=0
$$

for all $j>0$.

Proof This is a special case of [26, Corollary 4.1.9 (2)].

As an application of the above Bott type vanishing, we have the following:

Lemma 2.14 We have the following vanishing of cohomology groups:

(1) $H^{1}\left(G, \mathcal{T}_{G} \otimes \operatorname{Sym}^{k}\left(S^{\vee}\right)\right)=0$ for all $k \geq 0$.

(2) $H^{1}\left(G, S \otimes \operatorname{Sym}^{k}\left(S^{\vee}\right)\right)=0$ for all $k>0$.

(3) $H^{1}\left(G, \operatorname{Sym}^{k}\left(S^{\vee}\right)\right)=0$ for all $k>0$.

Proof We only prove the first assertion, since the other can be proved by similar computations. Recall that we have $\mathcal{T}_{G} \cong S^{\vee} \otimes Q$, and hence

$$
\mathcal{T}_{G} \otimes \operatorname{Sym}^{k}\left(S^{\vee}\right) \cong S^{\vee} \otimes \operatorname{Sym}^{k}\left(S^{\vee}\right) \otimes Q .
$$

Moreover, by Pieri formula (cf. [26, Corollary 2.3.5]), we have

$$
S^{\vee} \otimes \operatorname{Sym}^{k}\left(S^{\vee}\right) \cong K_{\nu_{1}}\left(S^{\vee}\right) \oplus K_{\nu_{2}}\left(S^{\vee}\right),
$$

where $v_{1}=(k, 1,0, \ldots, 0), v_{2}=(k+1,0, \ldots, 0) \in \mathbb{Z}^{\oplus i}$ (see also [26, Proposition 2.1.18] for the relation between the Schur and the Weyl functors). Hence we have

$$
\mathcal{T}_{G} \otimes \operatorname{Sym}^{k}\left(S^{\vee}\right) \cong V\left(\alpha_{1}\right) \oplus V\left(\alpha_{2}\right),
$$

where we put $\alpha_{t}=\left(v_{t}, 0, \ldots, 0,-1\right) \in \mathbb{Z}^{\oplus n}$ for $t=0,1$. Hence by Theorem 2.13, we obtain the vanishing $H^{1}\left(G, \mathcal{T}_{G} \otimes \operatorname{Sym}^{k}\left(S^{\vee}\right)\right)=0$ as required.

\section{Framed sheaves on the blow-up}

\subsection{ADHM description and wall crossing}

Let us consider the projective plane $\mathbb{P}^{2}=\mathbb{C}^{2} \cup l_{\infty}$ and its blow-up $f: \hat{\mathbb{P}}^{2} \rightarrow \mathbb{P}^{2}$ at the origin $0 \in \mathbb{C}^{2}$. Denote by $C \subset \mathbb{P}^{2}$ the $f$-exceptional curve. In this subsection, we recall the notion of $m$-stable sheaves on $\hat{\mathbb{P}}^{2}$ with framing at $l_{\infty}$ studied by Nakajima-Yoshioka [21]. 
Definition 3.1 Fix a non-negative integer $m \in \mathbb{Z}_{\geq 0}$. Let $(E, \Phi)$ be a framed sheaf on $\hat{\mathbb{P}}^{2}$, i.e., let $E$ be a coherent sheaf on $\hat{\mathbb{P}}^{2}$ and $\Phi:\left.E\right|_{l_{\infty}} \stackrel{\cong}{\rightrightarrows} \mathcal{O}_{l_{\infty}}^{\oplus \operatorname{ch}_{0}(E)}$ a framing at $l_{\infty}$. We say that $(E, \Phi)$ is $m$-stable if the following conditions hold:

(1) $\operatorname{Hom}\left(E(-m C), \mathcal{O}_{C}(-1)\right)=0$,

(2) $\operatorname{Hom}\left(\mathcal{O}_{C}, E(-m C)\right)=0$,

(3) $E$ is torsion free outside $C$.

Remark 3.2 Let $E$ be an $m$-stable framed sheaf. By [22, Proposition 1.9 (1)], the condition (1) in the above definition implies that the sheaf $E(-m C)$ is an object of the category $\operatorname{Per}\left(\hat{\mathbb{P}}^{2} / \mathbb{P}^{2}\right)$ of perverse coherent sheaves introduced by Bridgeland [6]. Hence we may also think of $m$-stable sheaves as stable objects in the category $\operatorname{Per}\left(\hat{\mathbb{P}}^{2} / \mathbb{P}^{2}\right) \otimes \mathcal{O}(m C)$.

We denote by $M^{m}(v)$ the fine moduli space of $m$-stable framed sheaves on $\hat{\mathbb{P}}^{2}$ with Chern character $v$. Let us recall the ADHM description of framed sheaves on $\hat{\mathbb{P}}^{2}$; Consider the following quiver $Q$

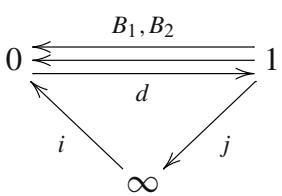

with the relation

$$
I: B_{1} d B_{2}-B_{2} d B_{1}+i j=0 .
$$

For a given Chern character

$$
v=\left(r,-k C, \operatorname{ch}_{2}\right) \in \bigoplus_{i=0}^{2} H^{2 i}\left(\hat{\mathbb{P}}^{2}, \mathbb{Q}\right),
$$

we associate the dimension vector $\vec{d}=\left(d_{0}, d_{1}, d_{\infty}\right)$ by the following formula:

$$
d_{\infty}=r, \quad k=d_{0}-d_{1}, \quad \operatorname{ch}_{2}=-\frac{1}{2}\left(d_{0}+d_{1}\right) .
$$

Theorem 3.3 [21] Let us fix a Chern character $v=\left(r,-k C, \operatorname{ch}_{2}\right) \in H^{2 *}\left(\hat{\mathbb{P}}^{2}, \mathbb{Q}\right)$ with $r>0, k \geq 0$, and associate the vector $\vec{d} \in \mathbb{Z}_{\geq 0}^{\oplus 3}$ as in (3.1). Then, in the region $\Omega:=$ $\left\{\left(\zeta_{0}, \zeta_{1}\right) \in \mathbb{R}^{2}: \zeta_{0}>0\right\}$ of King's stability parameters for $(Q, I)$-representations, the walls are classified as

$$
\mathcal{W}_{m}:=\left\{\left(\zeta_{0}, \zeta_{1}\right) \in \Omega: m \zeta_{0}+(m+1) \zeta_{1}=0\right\}, \quad m \in \mathbb{Z}_{\geq 0} .
$$

For $m \geq 1$, denote by $\mathcal{C}_{m}$ the chamber between the walls $\mathcal{W}_{m}$ and $\mathcal{W}_{m-1}$, and put $\mathcal{C}_{0}:=\left\{\left(\zeta_{0}, \zeta_{1}\right) \in \mathbb{R}^{2}: \zeta_{0}, \zeta_{1}<0\right\}$. The following statements hold:

(1) For each integer $m \in \mathbb{Z}_{\geq 0}$, and a stability condition $\zeta \in \mathcal{C}_{m}$, there exists an isomorphism $M^{m}(v) \cong M^{\zeta}(Q, I ; \vec{d})$. Here, $M^{\zeta}(Q, I ; \vec{d})$ denotes the moduli space of $\zeta$-stable $(Q$, I)-representations with dimension vector $\vec{d}$. Furthermore, these moduli spaces are either empty or smooth quasi-projective varieties of dimension $d_{\infty}\left(d_{0}+d_{1}\right)-\left(d_{0}-d_{1}\right)^{2}$. 
(2) We have a natural morphism

$$
M^{0}(v) \rightarrow M_{\mathbb{P}^{2}}\left(r, 0, \mathrm{ch}_{2}\right), \quad E \mapsto f_{*} E,
$$

which is an isomorphism when $k=0$. Here, $M_{\mathbb{P}^{2}}\left(r, 0, \mathrm{ch}_{2}\right)$ denotes the moduli space of torsion free framed sheaves on $\mathbb{P}^{2}$.

(3) There exists an integer $m_{0} \in \mathbb{Z}_{\geq 0}$ such that for every integer $m \geq m_{0}$, we have an isomorphism

$$
M^{m}(v) \cong M_{\hat{\mathbb{P}}^{2}}(v),
$$

where $M_{\hat{\mathbb{P}} 2}(v)$ denotes the moduli space of torsion free framed sheaves on $\hat{\mathbb{P}}^{2}$.

(4) For each integer $m \in \mathbb{Z}_{\geq 0}$ and a stability condition $\zeta \in \mathcal{W}_{m}$, there exists a set-theoretic bijection

$$
M^{\zeta}(Q, I ; \vec{d})=M^{m, m+1}(v):=\bigsqcup_{i \geq 0}\left(M^{m}\left(v-i c_{m}\right) \cap M^{m+1}\left(v-i c_{m}\right)\right),
$$

where we put $c_{m}:=\operatorname{ch}\left(\mathcal{O}_{C}(-m-1)\right)$.

Proof The classification of walls is explained in [21, Section 4.3]; The statements (1), (2), (3) are proved in Theorems 1.5 and 2.5, Proposition 7.4, and Proposition 7.1 in [21], respectively.

Let us now consider the statement (4). By [21, Section 4.3, Proposition 5.3], the wall $\mathcal{W}_{m}$ corresponds to the destabilizing object $\mathcal{O}_{C}(-m-1)$ in the following sense: for any stability condition $\zeta \in \mathcal{W}_{m}$ on the wall and any $\zeta$-semistable object $E$, its S-equivalence class is of the form $\mathcal{O}_{C}(-m-1)^{\oplus i} \oplus E^{\prime}$ for some non-negative integer $i \geq 0$ and $m$-stable and $(m+1)$-stable sheaf $E^{\prime}$ with $\operatorname{ch}\left(E^{\prime}\right)=v-i c_{m}$. Hence as a closed point in the moduli space, we have

$$
[E]=\left[\mathcal{O}_{C}(-m-1)^{\oplus i} \oplus E^{\prime}\right] \in M^{\zeta}(Q, I ; \vec{d}),
$$

i.e., the closed point $[E] \in M^{\zeta}(Q, I ; \vec{d})$ is uniquely determined by the sheaf $E^{\prime} \in M^{m}(v-$ $\left.i c_{m}\right) \cap M^{m+1}\left(v-i c_{m}\right)$. Hence we have the bijection as stated.

By the above theorem, we have the diagram as in (1.1) connecting the moduli spaces $M_{\mathbb{P}^{2}}\left(r, 0, \mathrm{ch}_{2}\right)$ and $M_{\hat{\mathbb{P}}^{2}}\left(r,-k C, \mathrm{ch}_{2}\right)$.

\subsection{Brill-Noether loci}

Next we recall the Brill-Noether stratifications on the moduli spaces and the determination of the fibers over $\xi_{m}^{ \pm}$. For each integer $i \in \mathbb{Z}_{\geq 0}$, let us consider the following locally closed subschemes:

$$
\begin{aligned}
& M^{m}(v)_{i}:=\left\{(E, \Phi) \in M^{m}(v): \operatorname{hom}\left(\mathcal{O}_{C}(-m-1), E\right)=i\right\}, \\
& M^{m+1}(v)_{i}:=\left\{(E, \Phi) \in M^{m+1}(v): \operatorname{hom}\left(E, \mathcal{O}_{C}(-m-1)\right)=i\right\} .
\end{aligned}
$$

We call them as the Brill-Noether strata. We also denote as $M^{m, m+1}(v)_{i}:=M^{m}\left(v-i c_{m}\right) \cap$ $M^{m+1}\left(v-i c_{m}\right)$.

Let us take an object $E \in M^{m}(v)$. By [22, Proposition 3.15], we have the exact sequence

$$
0 \rightarrow \operatorname{Hom}\left(\mathcal{O}_{C}(-m-1), E\right) \otimes \mathcal{O}_{C}(-m-1) \stackrel{e v}{\longrightarrow} E \rightarrow E^{\prime} \rightarrow 0
$$

for some object $E^{\prime} \in M^{m, m+1}(v)$. By (the proof of) Theorem $3.3(4)$, we have $\xi_{m}^{-}(E)=E^{\prime}$, where $\xi_{m}^{-}: M^{m}(v) \rightarrow M^{m, m+1}(v)$ is the morphism in the diagram(1.1). Hence the morphism 
$\xi_{m}^{-}$restricts to the morphism $\xi_{m, i}^{-}: M^{m}(v)_{i} \rightarrow M^{m, m+1}(v)_{i}$. Moreover, from the exact sequence (3.2), we see that an $m$-stable sheaf $E$ is $(m+1)$-stable if and only if the vanishing $\operatorname{Hom}\left(\mathcal{O}_{C}(-m-1), E\right)=0$ holds. Hence we have

$$
M^{m}(v)_{0}=M^{m}(v) \cap M^{m+1}(v) \subset M^{m}(v)
$$

and it is an open immersion.

Let us denote by

$$
\mathcal{E}_{i}^{\prime} \in \operatorname{Coh}\left(\hat{\mathbb{P}}^{2} \times M^{m, m+1}(v)_{i}\right)
$$

the universal family, and let

$$
p: \hat{\mathbb{P}}^{2} \times M^{m, m+1}(v)_{i} \rightarrow M^{m, m+1}(v)_{i}, \quad q: \hat{\mathbb{P}}^{2} \times M^{m, m+1}(v)_{i} \rightarrow \hat{\mathbb{P}}^{2}
$$

be the projections. The following theorem shows the structure of the morphism $\xi_{m}^{ \pm}$in terms of the Brill-Noether strata:

Theorem 3.4 [22, Proposition 3.31, Proposition 3.32] The morphisms

$$
\begin{aligned}
& \xi_{m, i}^{-}: M^{m}(v)_{i} \rightarrow M^{m, m+1}(v)_{i}, \\
& \xi_{m, i}^{+}: M^{m+1}(v)_{i} \rightarrow M^{m, m+1}(v)_{i}
\end{aligned}
$$

are identified with the morphisms

$$
\begin{aligned}
& \operatorname{Gr}\left(i, \mathcal{E} x t_{p}^{1}\left(\mathcal{E}_{i}^{\prime}, q^{*} \mathcal{O}_{C}(-m-1)\right)\right) \rightarrow M^{m, m+1}(v)_{i}, \\
& \operatorname{Gr}\left(i, \mathcal{E} x t_{p}^{1}\left(q^{*} \mathcal{O}_{C}(-m-1), \mathcal{E}_{i}^{\prime}\right)\right) \rightarrow M^{m, m+1}(v)_{i},
\end{aligned}
$$

respectively. In particular, every fiber of the morphisms $\xi_{m}^{ \pm}$is the Grassmannian variety.

\section{Birational geometry of moduli spaces}

In this section, we will prove that the diagram (1.1) realizes the MMP. The key ingredient is to compute the normal bundles of the fibers of $\xi_{m}^{ \pm}$, following the arguments of Ellingsrud and Göttsche [10] and Friedman and Qin [11]. We keep the notations as in the previous section. Fix integers $m, i \in \mathbb{Z}_{\geq 0}$. Let

$$
\mathcal{E}^{-} \in \operatorname{Coh}\left(\hat{\mathbb{P}}^{2} \times M^{m}(v)\right), \quad \mathcal{E}^{+} \in \operatorname{Coh}\left(\hat{\mathbb{P}}^{2} \times M^{m+1}(v)\right)
$$

be the universal families, and let

$$
\mathcal{W}_{i}^{-}:=\mathcal{E} x t_{p}^{1}\left(\mathcal{E}_{i}^{\prime}, q^{*} \mathcal{O}_{C}(-m-1)\right), \quad \mathcal{W}_{i}^{+}:=\mathcal{E} x t_{p}^{1}\left(q^{*} \mathcal{O}_{C}(-m-1), \mathcal{E}_{i}^{\prime}\right)
$$

be vector bundles on $M^{m, m+1}(v)_{i}$. We consider the Grassmannian bundles

$$
\begin{aligned}
& \pi^{-}: G_{i}^{-}:=\operatorname{Gr}\left(i, \mathcal{W}_{i}^{-}\right) \rightarrow M^{m, m+1}(v)_{i}, \\
& \pi^{+}: G_{i}^{+}:=\operatorname{Gr}\left(i, \mathcal{W}_{i}^{+}\right) \rightarrow M^{m, m+1}(v)_{i},
\end{aligned}
$$

which are isomorphic to the Brill-Noether loci $M^{m}(v)_{i}, M^{m+1}(v)_{i}$, respectively, by Theorem 3.4. On $G_{i}^{ \pm}$, we have the following tautological sequences:

$$
0 \rightarrow \mathcal{S}_{i}^{ \pm} \rightarrow \pi^{ \pm *} \mathcal{W}_{i}^{ \pm} \rightarrow \mathcal{Q}_{i}^{ \pm} \rightarrow 0
$$


Let $g^{ \pm}: \hat{\mathbb{P}}^{2} \times G_{i}^{ \pm} \rightarrow G_{i}^{ \pm}, h^{ \pm}: \hat{\mathbb{P}}^{2} \times G_{i}^{ \pm} \rightarrow \hat{\mathbb{P}}^{2}$ be the projections. We start with the following lemma.

Lemma 4.1 There exists an exact sequence

$$
\left.0 \rightarrow g^{-*} \mathcal{S}_{i}^{-\vee} \otimes h^{-*} \mathcal{O}_{C}(-m-1) \rightarrow \mathcal{E}^{-}\right|_{G_{i}^{-}} \rightarrow \pi_{X}^{-*} \mathcal{E}_{i}^{\prime} \rightarrow 0
$$

on $\operatorname{Coh}\left(\hat{\mathbb{P}}^{2} \times G_{i}^{-}\right)$. Similarly, we have

$$
\left.0 \rightarrow \pi_{X}^{+*} \mathcal{E}_{i}^{\prime} \rightarrow \mathcal{E}^{+}\right|_{G_{i}^{+}} \rightarrow g^{+*} \mathcal{S}_{i}^{+} \otimes h^{+*} \mathcal{O}(-m-1) \rightarrow 0 .
$$

on $\operatorname{Coh}\left(\hat{\mathbb{P}}^{2} \times G_{i}^{+}\right)$.

Proof Let us take an object $E^{\prime} \in M^{m, m+1}(v)_{i}$ and an $i$-dimensional subspace $V \subset$ $\operatorname{Ext}^{1}\left(E^{\prime}, \mathcal{O}_{C}(-m-1)\right)$. Then by [23, Proposition 4.7], the associated universal extension

$$
0 \rightarrow V^{\vee} \otimes \mathcal{O}_{C}(-m-1) \rightarrow E^{-} \rightarrow E^{\prime} \rightarrow 0
$$

defines an $m$-stable sheaf $E^{-} \in M^{m}(v)$. Hence $\left.\mathcal{E}^{-}\right|_{G_{i}^{-}}$coincides with the universal extension on $\hat{\mathbb{P}}^{2} \times G_{i}^{-}$, and the first assertion follows. The proof of the second assertion is similar.

\subsection{Birational geometry of moduli spaces}

The goal of this subsection is to prove the following:

Theorem 4.2 For any integers $m, i \in \mathbb{Z}_{\geq 0}$, we have isomorphisms

$$
\begin{aligned}
& \mathcal{N}_{G_{i}^{-} / M^{m}(v)} \cong \mathcal{S}_{i}^{-} \otimes \pi^{-*} \mathcal{W}_{i}^{+}, \\
& \mathcal{N}_{G_{i}^{+} / M^{m+1}(v)} \cong \mathcal{S}_{i}^{+} \otimes \pi^{+*} \mathcal{W}_{i}^{-} .
\end{aligned}
$$

See (4.2), (4.3), and (4.4) for the notations used in the above theorem. We divide the proof of the theorem into several lemmas.

Lemma 4.3 There exists a natural morphism

$$
\delta:\left.\mathcal{T}_{M^{m}(v)}\right|_{G_{i}^{-}} \rightarrow \mathcal{S}_{i}^{-} \otimes \pi^{-*} \mathcal{W}_{i}^{+} .
$$

Proof By the deformation-obstruction theory for framed sheaves (cf. [7, Theorem 4.3]), the tangent bundle of $M^{m}(v)$ is given as

$$
\mathcal{T}_{M^{m}(v)} \cong \mathcal{E} x t_{p_{M}}^{1}\left(\mathcal{E}^{-}, \mathcal{E}^{-}\left(-l_{\infty} \times M^{m}(v)\right)\right) .
$$

Now, applying the functor $\mathcal{H o m}_{g^{-}}\left(\left.\mathcal{E}^{-}\right|_{G_{i}^{-}},(-) \otimes \mathcal{O}\left(-l_{\infty} \times G_{i}^{-}\right)\right)$to the exact sequence (4.5), we get the morphism

$$
\delta_{1}: \mathcal{E} x t_{g^{-}}^{1}\left(\left.\mathcal{E}^{-}\right|_{G_{i}^{-}},\left.\mathcal{E}^{-}\right|_{G_{i}^{-}}\left(-l_{\infty} \times G_{i}^{-}\right)\right) \rightarrow \mathcal{E} x t_{g^{-}}^{1}\left(\left.\mathcal{E}^{-}\right|_{G_{i}^{-}}, \pi^{-*} \mathcal{E}_{i}^{\prime}\left(-l_{\infty} \times G_{i}^{-}\right)\right) .
$$

On the other hand, applying the functor $\mathcal{H}_{o m} m^{-}\left(-, \pi^{-*} \mathcal{E}_{i}^{\prime}\left(-l_{\infty} \times G_{i}^{-}\right)\right)$to the exact sequence (4.5), we get the morphism

$$
\begin{aligned}
\delta_{2}: \mathcal{E} x t_{g^{-}}^{1}\left(\left.\mathcal{E}^{-}\right|_{G_{i}^{-}}, \pi^{-*} \mathcal{E}_{i}^{\prime}\left(-l_{\infty} \times G_{i}^{-}\right)\right) \rightarrow \\
\quad \mathcal{E} x t_{g^{-}}^{1}\left(g^{-*} \mathcal{S}_{i}^{-\vee} \otimes h^{-*} \mathcal{O}_{C}(-m-1), \pi^{-*} \mathcal{E}_{i}^{\prime}\right),
\end{aligned}
$$


since $\mathcal{O}_{C}\left(l_{\infty}\right)=\mathcal{O}_{C}$. Note that we have

$$
\mathcal{E} x t_{g^{-}}^{1}\left(g^{-*} \mathcal{S}_{i}^{-\vee} \otimes h^{-*} \mathcal{O}_{C}(-m-1), \pi^{-*} \mathcal{E}_{i}^{\prime}\right) \cong \mathcal{S}_{i}^{-} \otimes \pi^{-*} \mathcal{W}_{i}^{+} .
$$

By the above arguments, we have a morphism

$$
\delta:=\delta_{2} \circ \delta_{1}:\left.\mathcal{T}_{M^{m}(v)}\right|_{G^{-}} \rightarrow \mathcal{S}_{i}^{-} \otimes \pi^{-*} \mathcal{W}_{i}^{+} .
$$

In the following lemma, we show that our morphism $\delta$ is surjective, by checking it on the fibers of the morphism $\pi^{-}: G_{i}^{-} \rightarrow M^{m, m+1}(v)_{i}$ in (4.3). By abuse of notation, we also denote by $\delta_{1}, \delta_{2}$ their restrictions to the $\pi^{-}$-fibers.

Lemma 4.4 Let us take an object $E^{\prime} \in M^{m, m+1}(v)_{i}$ and put $W^{-}:=\operatorname{Ext}^{1}\left(E^{\prime}, \mathcal{O}_{C}(-m-1)\right)$, $W^{+}:=\operatorname{Ext}^{1}\left(\mathcal{O}_{C}(-m-1), E^{\prime}\right)$. Take also an $i$-dimensional subspace $V \subset W^{-}$, and let $E^{-} \in M^{m}(v)$ be the associated universal extension. Then we have the following exact sequences:

$$
\begin{aligned}
& \operatorname{Hom}\left(V, W^{-} / V\right) \stackrel{\alpha_{1}}{\rightarrow} \operatorname{Ext}^{1}\left(E^{-}, E^{-}\left(-l_{\infty}\right)\right) \stackrel{\delta_{1}}{\rightarrow} \operatorname{Ext}^{1}\left(E^{-}, E^{\prime}\left(-l_{\infty}\right)\right) \rightarrow 0, \\
& \operatorname{Ext}^{1}\left(E^{\prime}, E^{\prime}\left(-l_{\infty}\right)\right) \stackrel{\alpha_{2}}{\rightarrow} \operatorname{Ext}^{1}\left(E^{-}, E^{\prime}\left(-l_{\infty}\right)\right) \stackrel{\delta_{2}}{\rightarrow} V \otimes W^{+} \rightarrow 0 .
\end{aligned}
$$

Proof By replacing the object $E^{\prime} \in M^{m, m+1}(v)_{i}$ with $E^{\prime} \otimes \mathcal{O}_{\hat{\mathbb{P}}^{2}}(-m C) \in M^{m, m+1}\left(v \cdot e^{-m C}\right)_{i}$, we may assume $m=0$. Let us take an object $E^{\prime} \in M^{0,1}(v)_{i}$ and an $i$-dimensional subspace $V \subset \operatorname{Ext}^{1}\left(E^{\prime}, \mathcal{O}_{C}(-1)\right)$. Let

$$
0 \rightarrow V^{\vee} \otimes \mathcal{O}_{C}(-1) \rightarrow E^{-} \rightarrow E^{\prime} \rightarrow 0
$$

be the associated universal extension. By applying the functor $\operatorname{Hom}\left(E^{-},(-) \otimes \mathcal{O}\left(-l_{\infty}\right)\right)$, we have the exact sequence

$$
\begin{aligned}
& \operatorname{Ext}^{1}\left(E^{-}, V^{\vee} \otimes \mathcal{O}_{C}(-1)\right) \rightarrow \operatorname{Ext}^{1}\left(E^{-}, E^{-}\left(-l_{\infty}\right)\right) \stackrel{\delta_{1}}{\rightarrow} \operatorname{Ext}^{1}\left(E^{-}, E^{\prime}\left(-l_{\infty}\right)\right) \\
\rightarrow & \operatorname{Ext}^{2}\left(E^{-}, V^{\vee} \otimes \mathcal{O}_{C}(-1)\right) .
\end{aligned}
$$

Note that we have used the fact that $\mathcal{O}_{C}\left(-l_{\infty}\right)=\mathcal{O}_{C}$. By Serre duality and the 0 -stability of $E^{-}$, we have the vanishing

$$
\operatorname{Ext}^{2}\left(E^{-}, \mathcal{O}_{C}(-1)\right) \cong \operatorname{Hom}\left(\mathcal{O}_{C}, E^{-}\right)^{\vee}=0
$$

and hence $\delta_{1}$ is surjective. Furthermore, by applying the functor $\operatorname{Hom}\left(-, \mathcal{O}_{C}(-1)\right)$ to the exact sequence (4.7) we have the exact sequence

$$
0 \rightarrow V \rightarrow W^{-} \rightarrow \operatorname{Ext}^{1}\left(E^{-}, \mathcal{O}_{C}(-1)\right) \rightarrow \operatorname{Ext}^{1}\left(V^{\vee} \otimes \mathcal{O}_{C}(-1), \mathcal{O}_{C}(-1)\right)=0 .
$$

Hence we have

$$
\operatorname{Ext}^{1}\left(E^{-}, V^{\vee} \otimes \mathcal{O}_{C}(-1)\right) \cong \operatorname{Hom}\left(V, W^{-} / V\right)
$$

as required.

Similarly, applying the functor $\operatorname{Hom}\left(-, E^{\prime}\left(-l_{\infty}\right)\right)$ to the exact sequence $(4.7)$, we obtain

$$
\begin{aligned}
& \operatorname{Ext}^{1}\left(E^{\prime}, E^{\prime}\left(-l_{\infty}\right)\right) \stackrel{\alpha_{2}}{\rightarrow} \operatorname{Ext}^{1}\left(E^{-}, E^{\prime}\left(-l_{\infty}\right)\right) \stackrel{\delta_{2}}{\rightarrow} V \otimes W^{+} \\
& \quad \rightarrow \operatorname{Ext}^{2}\left(E^{\prime}, E^{\prime}\left(-l_{\infty}\right)\right) .
\end{aligned}
$$

By Lemma 4.5 below, we have the vanishing $\operatorname{Ext}^{2}\left(E^{\prime}, E^{\prime}\left(-l_{\infty}\right)\right)=0$ and hence $\delta_{2}$ is surjective. 
Lemma 4.5 Let $F$ be a 0 -stable sheaf. Then we have the vanishing

$$
\operatorname{Ext}^{2}\left(F, F\left(-l_{\infty}\right)\right)=0 .
$$

Proof By Serre duality, we have

$$
\operatorname{Ext}^{2}\left(F, F\left(-l_{\infty}\right)\right) \cong \operatorname{Hom}\left(F\left(-l_{\infty}\right), F \otimes \omega_{\hat{\mathbb{P}}^{2}}\right)^{\vee} .
$$

Moreover, as in the proof of [22, Lemma 3.6], we have an injection

$$
\operatorname{Hom}_{\hat{\mathbb{P}}^{2}}\left(F\left(-l_{\infty}\right), F \otimes \omega_{\hat{\mathbb{P}}^{2}}\right) \hookrightarrow \operatorname{Hom}_{\mathbb{P}^{2}}\left(\left(f_{*} F\right)^{\vee \vee}\left(-l_{\infty}\right),\left(f_{*} F\right)^{\vee \vee} \otimes \omega_{\mathbb{P}^{2}}\right),
$$

where $f: \hat{\mathbb{P}}^{2} \rightarrow \mathbb{P}^{2}$ is the blow-up morphism. Putting $G:=\left(f_{*} F\right)^{\vee \vee}$, it is enough to show

$$
\operatorname{Hom}\left(G\left(-l_{\infty}\right), G \otimes \omega_{\mathbb{P}^{2}}\right)=\operatorname{Hom}(G, G(-2))=0 .
$$

By applying the functor $\operatorname{Hom}(G,-)$ to the exact sequence

$$
\left.0 \rightarrow G(-3) \rightarrow G(-2) \rightarrow G(-2)\right|_{l_{\infty}} \rightarrow 0
$$

we obtain

$$
0 \rightarrow \operatorname{Hom}(G, G(-3)) \rightarrow \operatorname{Hom}(G, G(-2)) \rightarrow \operatorname{Hom}\left(G,\left.G(-2)\right|_{l_{\infty}}\right)
$$
have

On the other hand, as $G$ is a framed sheaf on $\mathbb{P}^{2}$, we have $\left.G\right|_{l_{\infty}} \cong \mathcal{O}_{\mathbb{P}^{2}}^{\oplus \operatorname{ch}_{0}(G)}$. Hence we

$$
\begin{aligned}
\operatorname{Hom}\left(G,\left.G(-2)\right|_{l_{\infty}}\right) & \cong \operatorname{Hom}\left(\left.G\right|_{l_{\infty}},\left.G(-2)\right|_{l_{\infty}}\right) \\
& \cong \operatorname{Hom}\left(\mathcal{O}_{l_{\infty}}, \mathcal{O}_{l_{\infty}}(-2)\right)^{\oplus \operatorname{ch}_{0}(G)^{2}} \\
& =0 .
\end{aligned}
$$

Combining with the exact sequence (4.9), we conclude that

$$
\operatorname{Hom}(G, G(-2)) \cong \operatorname{Hom}(G, G(-3)) .
$$

By tensoring the exact sequence (4.8) with $\mathcal{O}_{\mathbb{P}^{2}}(-i)$ and repeating the above argument, we can inductively prove the isomorphism

$$
\operatorname{Hom}(G, G(-2)) \cong \operatorname{Hom}(G, G(-i-3))
$$

for all $i \geq 0$. By Serre duality, we obtain

$$
\operatorname{Hom}(G, G(-i-3)) \cong H^{2}\left(G \otimes G^{\vee}(i)\right)^{\vee},
$$

and the right hand side vanishes for sufficiently large $i>0$, since $\mathcal{O}_{\mathbb{P}^{2}}(1)$ is ample. We conclude that $\operatorname{Hom}(G, G(-2))=0$ as required.

We also need the following:

Lemma 4.6 We have the equalities

$$
\begin{aligned}
& \operatorname{dim} M^{m}(v)=\operatorname{dim} G_{i}^{-}+\operatorname{rk}\left(\mathcal{S}_{i}^{-} \otimes \pi^{-*} \mathcal{W}_{i}^{+}\right), \\
& \operatorname{dim} M^{m+1}(v)=\operatorname{dim} G_{i}^{+}+\operatorname{rk}\left(\mathcal{S}_{i}^{+} \otimes \pi^{+*} \mathcal{W}_{i}^{-}\right) .
\end{aligned}
$$


Proof We only prove the first equality. By the dimension formula for the framed moduli space in Theorem 3.3 (1), we have

$$
\begin{aligned}
\operatorname{dim} M^{m}(v) & =d_{\infty}\left(d_{0}+d_{1}\right)-\left(d_{0}-d_{1}\right)^{2} \\
& =-2 r \mathrm{ch}_{2}-k^{2},
\end{aligned}
$$

where the vector $\left(d_{0}, d_{1}, d_{\infty}\right)$ is defined as in (3.1). On the other hand, for any object $E^{\prime} \in$ $M^{m, m+1}(v)_{i}$, we have

$$
\begin{aligned}
\operatorname{dim} G_{i}^{-} & =\operatorname{dim} M^{m, m+1}(v)_{i}+i\left(\operatorname{ext}^{1}\left(E^{\prime}, \mathcal{O}_{C}(-m-1)\right)-i\right) \\
& =\operatorname{dim} M^{m}\left(v-i c_{m}\right)-i\left(\chi\left(E^{\prime}, \mathcal{O}_{C}(-m-1)\right)+i\right),
\end{aligned}
$$

where the first equality follows from the fact that $\pi^{-}: G_{i}^{-} \rightarrow M^{m, m+1}(v)_{i}$ is a Grassmannian bundle defined as in (4.3). For the second equality, first observe that we have $\chi\left(E^{\prime}, \mathcal{O}_{C}(-m-\right.$ $1))=-\operatorname{ext}^{1}\left(E^{\prime}, \mathcal{O}_{C}(-m-1)\right)$ since $E^{\prime}$ is $m$-stable and $(m+1)$-stable. Moreover, the inclusion

$$
M^{m, m+1}(v)_{i}=M^{m}\left(v-i c_{m}\right) \cap M^{m+1}\left(v-i c_{m}\right) \subset M^{m}\left(v-i c_{m}\right)
$$

is an open immersion and hence the second equality in (4.11) holds. Similarly, by using the $m$-stability and $(m+1)$-stability of $E^{\prime}$, we have

$$
\begin{aligned}
\operatorname{rk}\left(\mathcal{S}_{i}^{-} \otimes \pi^{-*} \mathcal{W}_{i}^{+}\right) & =i \operatorname{ext}^{1}\left(\mathcal{O}_{C}(-m-1), E^{\prime}\right) \\
& =-i \cdot \chi\left(\mathcal{O}_{C}(-m-1), E^{\prime}\right)
\end{aligned}
$$

(see (4.2) and (4.4) for the definitions of $\mathcal{W}_{i}^{+}$and $\mathcal{S}_{i}^{-}$). Combining the equalities (4.10), (4.11) and (4.12), we obtain

$$
\begin{aligned}
\operatorname{dim} G_{i}^{-}+\operatorname{rk}\left(\mathcal{S}_{i}^{-} \otimes \pi^{-*} \mathcal{W}_{i}^{+}\right) \\
=\operatorname{dim} M^{m}\left(v-i c_{m}\right)-i\left(i+\chi\left(E^{\prime}, \mathcal{O}_{C}(-m-1)\right)+\chi\left(\mathcal{O}_{C}(-m-1), E^{\prime}\right)\right) \\
=-2 r\left(\operatorname{ch}_{2}+i\left(m+\frac{1}{2}\right)\right)-(k+i)^{2} \\
\quad-i^{2}-i\left(\chi\left(E^{\prime}, \mathcal{O}_{C}(-m-1)\right)+\chi\left(\mathcal{O}_{C}(-m-1), E^{\prime}\right)\right) \\
=-2 r \operatorname{ch}_{2}-k^{2}=\operatorname{dim} M^{m}(v),
\end{aligned}
$$

where the second equality holds since we have

$$
v-i c_{m}=v-i \operatorname{ch}\left(\mathcal{O}_{C}(-m-1)\right)=\left(r,-(k+i) C, \operatorname{ch}_{2}+i\left(m+\frac{1}{2}\right)\right),
$$

and for the third equality, we use the Serre duality $\chi\left(E^{\prime}, \mathcal{O}_{C}(-m-1)\right)=\chi\left(\mathcal{O}_{C}(-m), E^{\prime}\right)$, together with the Riemann-Roch theorem

$$
-\chi\left(\mathcal{O}_{C}(-m), E^{\prime}\right)=r m+k+i .
$$

Now we begin the proof of Theorem 4.2.

Proof of Theorem 4.2 We only prove the first assertion. By Lemmas 4.3 and 4.4, we have a surjective morphism

$$
\delta:\left.\mathcal{T}_{M^{m}(v)}\right|_{G_{i}^{-}} \rightarrow \mathcal{S}_{i}^{-} \otimes \pi^{-*} \mathcal{W}_{i}^{+}
$$


We need to show the isomorphism

$$
\mathcal{T}_{G_{i}^{-}} \cong \operatorname{ker}(\delta) \text {. }
$$

Let the notations be as in Lemma 4.4. By Lemma 4.6, the vector spaces $T_{E^{-}} G_{i}^{-}$and $\operatorname{ker}\left(\delta_{E^{-}}\right)$ are of the same dimension. Hence it is enough to show that the composition

$$
T_{E^{-}} G_{i}^{-} \hookrightarrow T_{E^{-}} M^{m}(v) \stackrel{\delta_{E^{-}}}{\longrightarrow} \operatorname{Ext}^{1}\left(V \otimes \mathcal{O}_{C}(-m-1), E^{\prime}\right)
$$

is zero. Indeed, if this is the case, then we have $T_{E^{-}} G_{i}^{-}=\operatorname{ker}\left(\delta_{E^{-}}\right)$, which induces a surjection $\mathcal{T}_{G_{i}^{-}} \rightarrow \operatorname{ker}(\delta)$ between torsion free sheaves of the same rank: it should be an isomorphism.

We have the exact sequence

$$
0 \rightarrow \operatorname{Hom}\left(V, W^{-} / V\right) \rightarrow T_{E^{-}} G_{i}^{-} \rightarrow T_{E^{\prime}} M^{m, m+1}(v)_{i} \rightarrow 0 .
$$

We can see that the composition

$$
\operatorname{Hom}\left(V, W^{-} / V\right) \rightarrow T_{E^{-}} G_{i}^{-} \rightarrow T_{E^{-}} M^{m}(v)
$$

coincides with the morphism $\alpha_{1}$ in the exact sequence (4.6). In particular, it becomes zero after composing with $\delta_{1}$. Hence the morphism

$$
T_{E^{-}} G_{i}^{-} \hookrightarrow T_{E^{-}} M^{m}(v) \stackrel{\delta}{\rightarrow} V \otimes W^{+}
$$

factors through $T_{E^{\prime}} M^{m, m+1}(v)_{i}$. Similarly, the morphism $T_{E^{\prime}} M^{m, m+1}(v)_{i} \rightarrow V \otimes W^{+}$ coincides with $\delta \circ \alpha_{2}$, which is zero by the second exact sequence in (4.6). We conclude that $T_{E^{-}} G_{i}^{-}=\operatorname{ker}\left(\delta_{E^{-}}\right)$as required.

Now we have the following theorem:

Theorem 4.7 Fix a Chern character of the form $v=\left(r, 0, \mathrm{ch}_{2}\right) \in H^{2 *}\left(\hat{\mathbb{P}}^{2}, \mathbb{Q}\right)$. Then the diagram (1.1) is a minimal model program for the moduli space $M_{\hat{\mathbb{P}}^{2}}(v)$ of framed torsion free sheaves on the blow-up $\hat{\mathbb{P}}^{2}$. The program ends with the minimal model, the moduli space $M_{\mathbb{P}^{2}}\left(r, 0, \mathrm{ch}_{2}\right)$ of framed torsion free sheaves on $\mathbb{P}^{2}$, which is a hyper-Kähler manifold.

Proof We claim that for each $m \in \mathbb{Z}_{\geq 0}$, the morphism $\xi_{m}^{+}$(resp. $\xi_{m}^{-}$) is a $K$-negative (resp. $K$-positive) contraction (cf. Definition 2.1). By Lemma 2.9 and Theorem 4.2, it is enough to show the inequality

$$
\operatorname{rk} \mathcal{W}_{i}^{+}>\mathrm{rk}_{i}^{-}
$$

(see (4.2) for the difinitions of $\mathcal{W}_{i}^{ \pm}$), which is equivalent to the inequality

$$
\operatorname{ext}^{1}\left(\mathcal{O}_{C}(-m-1), E^{\prime}\right)>\operatorname{ext}^{1}\left(E^{\prime}, \mathcal{O}_{C}(-m-1)\right) .
$$

Now the assertion directly follows from the Riemann-Roch theorem. Explicitly, we have

$$
\begin{aligned}
& \operatorname{ext}^{1}\left(\mathcal{O}_{C}(-m-1), E^{\prime}\right)-\operatorname{ext}^{1}\left(E^{\prime}, \mathcal{O}_{C}(-m-1)\right) \\
& =\chi\left(E^{\prime}, \mathcal{O}_{C}(-m-1)\right)-\chi\left(\mathcal{O}_{C}(-m-1), E^{\prime}\right) \\
& =r .
\end{aligned}
$$

To see that the moduli space $M_{\mathbb{P}^{2}}\left(r, 0, \mathrm{ch}_{2}\right)$ is hyper-Kähler, recall that the space $M_{\mathbb{P}^{2}}\left(r, 0, \mathrm{ch}_{2}\right)$ is isomorphic to Nakajima's quiver variety associated with the quiver with one vertex and one loop [20, Chapter 2]. By the general fact that Nakajima's quiver varieties are hyper-Kähler [12,19], so is the variety $M_{\mathbb{P}^{2}}\left(r, 0, \mathrm{ch}_{2}\right)$. 
From the arguments above, we can also deduce the following result:

Proposition 4.8 Let us take a Chern character $v=\left(r, 0, \mathrm{ch}_{2}\right) \in H^{2 *}\left(\hat{\mathbb{P}}^{2}, \mathbb{Q}\right)$. The following statements hold.

(1) For every integer $m \geq 1$, the morphism $\xi_{m}^{ \pm}$is a small contraction.

(2) The morphism $\xi_{0}^{+}$is a divisorial contraction.

Proof By Lemma 4.6 and Eq. (4.13), we have the inequality $\operatorname{dim} G_{i}^{+}>\operatorname{dim} G_{i}^{-}$. Hence it is enough to estimate the dimension of $G_{i}^{+}$. By Lemma 4.6, we have

$$
\begin{aligned}
\operatorname{codim}\left(G_{i}^{+}, M^{m+1}(v)\right) & =\operatorname{rk}\left(\mathcal{S}_{i}^{+} \otimes \pi^{+*} \mathcal{W}_{i}^{-}\right) \\
& =i \operatorname{ext}^{1}\left(E^{\prime}, \mathcal{O}_{C}(-m-1)\right)=r i m+i^{2} .
\end{aligned}
$$

It follows that for each $m \geq 1$, the morphism $\xi_{m}^{ \pm}$is a small contraction, while $\xi_{0}^{+}$is a divisorial contraction.

\subsection{Fully faithful embedding between derived categories}

As an application of Theorem 4.2, we show the existence of the fully faithful embedding between the derived categories of the moduli spaces $M^{m}(v), M^{m+1}(v)$. Let $W^{m}:=$ $M^{m}(v) \times_{M^{m, m+1}(v)} M^{m+1}(v)$ be the fiber product.

Theorem 4.9 Let us fix a Chern character $v=\left(r, 0, \mathrm{ch}_{2}\right) \in H^{2 *}\left(\hat{\mathbb{P}}^{2}, \mathbb{Q}\right)$. Then for each integer $m \in \mathbb{Z}_{\geq 0}$, we have the fully faithful functor

$$
\Phi=\Phi^{\mathcal{O}_{W^{m}}}: D^{b}\left(M^{m}(v)\right) \hookrightarrow D^{b}\left(M^{m+1}(v)\right)
$$

whose Fourier-Mukai kernel is $\mathcal{O}_{W^{m}}$. In particular, we have a fully faithful embedding

$$
D^{b}\left(M_{\mathbb{P}^{2}}\left(r, 0, \mathrm{ch}_{2}\right)\right) \hookrightarrow D^{b}\left(M_{\hat{\mathbb{P}}^{2}}\left(r, 0, \mathrm{ch}_{2}\right)\right) .
$$

Proof By Lemma 2.8, we can reduce the statement to the formal completion at a point $\left[E^{\prime}\right] \in M^{m, m+1}(v)$.

We claim that the diagram (1.1) is formally locally isomorphic to the Grassmannian flip appered in Sect. 2.3, by using Lemma 2.8. Let us take an object $\left[E^{\prime}\right] \in M^{m, m+1}(v)_{i}$ and put $U:=\operatorname{Ext}^{1}\left(E^{\prime}\left(-l_{\infty}\right), E^{\prime}\right), W^{-}:=\operatorname{Ext}^{1}\left(E^{\prime}, \mathcal{O}_{C}(-m-1)\right), W^{+}:=\operatorname{Ext}^{1}\left(\mathcal{O}_{C}(-m-1), E^{\prime}\right)$. Recall that the fibers of $\xi_{m}^{ \pm}$are the Grassmannian varieties

$$
\left(\xi_{m}^{ \pm}\right)^{-1}\left(\left[E^{\prime}\right]\right)=G^{ \pm}\left(E^{\prime}\right):=\operatorname{Gr}\left(i, W^{ \pm}\right) .
$$

By Theorem 4.2, their normal bundles are given as

$$
\begin{aligned}
& N_{G^{-}\left(E^{\prime}\right) / M^{m}(v)}=S^{-} \otimes W^{+} \oplus \mathcal{O}^{\oplus \operatorname{dim} U}, \\
& N_{G^{+}\left(E^{\prime}\right) / M^{m+1}(v)}=S^{+} \otimes W^{-} \oplus \mathcal{O}^{\oplus \operatorname{dim} U} .
\end{aligned}
$$

Here, $S^{ \pm}$denotes the tautological subbundles on $G^{ \pm}\left(E^{\prime}\right)$. First note that the conormal bundle $N_{G^{+}\left(E^{\prime}\right) / M^{m+1}(v)}^{\vee}$ is nef as it is globally generated. Combining with Lemma 2.14, we can apply Lemma 2.8 to $G^{+}(E) \subset M^{m+1}(v)$. Moreover, its flip is unique by [16, Corollary 6.4]. We conclude that the formal completion of the diagram (1.1) is isomorphic to the formal 
completion of the diagram

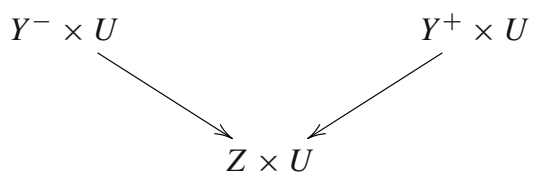

at a point $(0,0) \in Z \times U$. Here, varieties $Y^{ \pm}$and $Z$ are defined as

$$
\begin{aligned}
& Y^{-}:=\operatorname{Tot}_{G^{-}\left(E^{\prime}\right)}\left(S^{-} \otimes W^{+}\right), \\
& Y^{+}:=\operatorname{Tot}_{G^{+}\left(E^{\prime}\right)}\left(S^{+} \otimes W^{-}\right), \\
& Z:=\left\{a \in \operatorname{Hom}\left(\left(W^{-}\right)^{\vee}, W^{+}\right): \operatorname{rk} a \leq i\right\}
\end{aligned}
$$

(see Sect. 2.3). By Theorem 2.10, we have the fully faithful functor

$$
\Phi^{l o c}: D^{b}\left(Y^{-}\right) \hookrightarrow D^{b}\left(Y^{+}\right)
$$

whose Fourier-Mukai kernel is the structure sheaf of the fiber product $Y^{-} \times_{Z} Y^{+}$. Hence globally, the functor $\Phi: D^{b}\left(M^{m}(v)\right) \rightarrow D^{b}\left(M^{m+1}(v)\right)$ is fully faithful.

\subsection{Projective case}

Let $S$ be a smooth projective surface, $f: \hat{S} \rightarrow S$ be the blow-up at a point. Let $H$ be an ample divisor on $S$. In this setting, we can consider the $m$-stability for coherent sheaves $E$ on $\hat{S}$ (cf. [22]), by replacing the condition (3) in Definition 3.1 with

(3)' $f_{*}(E(-m C))$ is $\mu_{H}$-stable.

Let us fix a cohomology class $w=\left(w_{0}, w_{1}, w_{2}\right) \in H^{2 *}(S, \mathbb{Q})$ which is in the image of the Chern character map, and $v:=f^{*} w \in H^{2 *}(\hat{S}, \mathbb{Q})$. Assume the following conditions hold:

- $K_{S} \cdot H<0$,

- $w_{0}>0$,

- $\operatorname{gcd}\left(w_{0}, H \cdot w_{1}\right)=1$.

Then by [22, Corollary 3.7], the moduli space $M^{m}(v)$ of $m$-stable sheaves with Chern character $v$ is smooth. Moreover, the analogous results as Theorems 3.3 and 3.4 hold. More precisely, the moduli spaces $M^{m}(v)$ satisfy the following properties:

- $M^{m}(v)$ has the Brill-Noether stratification as in Sect. 3.2 by [22, Propositions 3.31 and 3.32].

- For $m=0$, we have an isomorphism $f_{*}: M^{0}(v) \stackrel{\cong}{\rightrightarrows} M_{H}(w)$, where $M_{H}(v)$ denotes the moduli space of Gieseker stable sheaves on $S$ with respect to the polarization $H$, by [22, Proposition 3.3].

- For $m \gg 0$, we have an isomorphism $M^{m}(v) \cong M_{f^{*} H-\epsilon C}(v)$, where $M_{f^{*} H-\epsilon C}(v)$ denotes the moduli space of Gieseker stable sheaves on $\hat{S}$ with respect to the polarization $f^{*} H-\epsilon C$ for sufficiently small $\epsilon>0$, by [22, Proposition 3.37].

In the projective setting, we can also prove the results in the previous subsections by using the properties listed above. We omit the proof, since the arguments are very similar. 
Theorem 4.10 Let the notations be as above. Take a sufficiently small positive real number $0<\epsilon \ll 1$. Then an MMP for the moduli space $M_{f^{*} H-\epsilon C}(v)$ of Gieseker stable sheaves on $\hat{S}$ is reduced to an MMP for the moduli $M_{H}(w)$ on $S$. Furthermore, there exists a fully faithful embedding

$$
D^{b}\left(M_{H}(w)\right) \hookrightarrow D^{b}\left(M_{f^{*} H-\epsilon C}(v)\right)
$$

between their derived categories.

\section{Examples}

In this section, we give some explicit examples.

\subsection{Simplest example}

As the first example, we consider the case when the Chern character is $(r, 0,-1)$ with $r \geq 1$. In this case, the corresponding quiver representation is

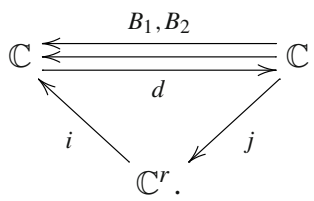

We begin with the following easy observation:

Lemma 5.1 The moduli spaces $M^{m}(r, 0,-1)$ and $M^{m+1}(r, 0,-1)$ are isomorphic for $m \geq$ 1.

Proof Let $m \geq 0$ and $E \in M^{m}(r, 0,-1)$ be an $m$-stable sheaf. Assume that $E$ is not $(m+1)$ stable. Then we must have $\operatorname{Hom}\left(\mathcal{O}_{C}(-m-1), E\right) \neq 0$, and obtain the exact sequence

$$
0 \rightarrow \operatorname{Hom}\left(\mathcal{O}_{C}(-m-1), E\right) \otimes \mathcal{O}_{C}(-m-1) \stackrel{e v}{\longrightarrow} E \rightarrow E^{\prime} \rightarrow 0
$$

as in (3.2), for some $m$-stable and $(m+1)$-stable sheaf $E^{\prime}$.

On the other hand, by [21, Proposition 5.3], the sheaf $\mathcal{O}_{C}(-m-1)$ corresponds to the quiver representation

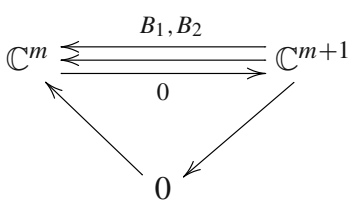

with $B_{1}=\left(1_{m}, 0\right), B_{2}=\left(0,1_{m}\right)$, where $1_{m}$ denotes the $(m \times m)$ identity matrix. Hence the representation (5.2) cannot have an injection into the representation (5.1) for $m>0$. This shows the inclusion $M^{m}(r, 0,-1) \subset M^{m+1}(r, 0,-1)$ for $m>0$. A similar argument shows that the opposite inclusion $M^{m+1}(r, 0,-1) \subset M^{m}(r, 0,-1)$.

Furthermore, we can describe these moduli spaces explicitly: 
Lemma 5.2 We have the diagram

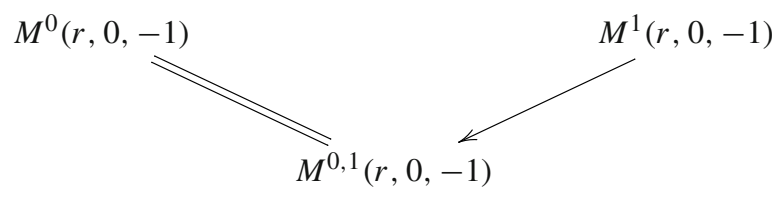

and isomorphisms

$$
\begin{aligned}
& M_{\mathbb{P}^{2}}(r, 0,-1) \cong M^{0}(r, 0,-1) \cong \operatorname{Tot}_{\mathbb{P}^{(r-1)}}\left(\mathcal{O}^{\oplus 2} \oplus \Omega\right), \\
& M_{\hat{\mathbb{P}}^{2}}(r, 0,-n) \cong M^{1}(r, 0,-1) \cong \mathrm{Bl}_{\mathbb{P}^{(r-1)}} M^{0}(r, 0,-1),
\end{aligned}
$$

where $\mathbb{P}^{(r-1)}$ is embedded into $M^{0}(r, 0,-1)$ as the zero section.

Note that when $r=1$, we recover the blow-up morphism $\hat{\mathbb{C}}^{2} \rightarrow \mathbb{C}^{2}$ as the moduli spaces (cf. [21, Theorem 2.16]).

Before starting the proof, let us recall the ADHM description of the framed sheaves on $\mathbb{P}^{2}$ (see [21, Section 1] and [20, Capter 2] for the details). Let $V, W$ be vector spaces. An $A D H M$ data is the data $X:=\left(B_{1}, B_{2}, i, j\right)$, where $B_{\alpha} \in \operatorname{End}(V), i \in \operatorname{Hom}(W, V)$, and $j \in \operatorname{Hom}(V, W)$ satisfying the relation

$$
\left[B_{1}, B_{2}\right]+i j=0 \text {. }
$$

An ADHM data $X=\left(B_{1}, B_{2}, i, j\right)$ is called stable if there is no proper subspace $T \subset V$ such that $B_{\alpha}(T) \subset T$ for $\alpha=1,2$, and Image $(i) \subset T$. We have the moduli space of stable ADHM data as the quotient of the stable locus inside the affine space

$$
\operatorname{End}(V)^{\times 2} \times \operatorname{Hom}(W, V) \times \operatorname{Hom}(V, W)
$$

modulo the natural GL( $V)$-action.

Then the moduli space $M_{\mathbb{P}^{2}}\left(r, 0, \mathrm{ch}_{2}\right)$ of torsion free framed sheaves on $\mathbb{P}^{2}$ is isomorphic to the moduli space of stable ADHM data with $\operatorname{dim} V=-\mathrm{ch}_{2}, \operatorname{dim} W=r$ (cf. [20, Chapter 2])

Proof of Lemma 5.2 Let us put $\operatorname{dim} V=1, \operatorname{dim} W=r$, and let $X=\left(B_{1}, B_{2}, i, j\right)$ be a stable ADHM data. Since $\operatorname{dim} V=1$, the relation (5.3) becomes $i j=0$, and the stability condition becomes $i \neq 0$. Hence the stable locus is given as

$$
\left.\left(\operatorname{End}(V)^{\times 2} \times\left(W^{\vee} \backslash\{0\}\right) \times W\right)\right) \cap \mu^{-1}(0),
$$

where

$$
\mu: \operatorname{End}(V)^{\times 2} \times W^{\vee} \times W \rightarrow \mathbb{C}, \quad\left(B_{1}, B_{2}, i, j\right) \mapsto i j
$$

is the moment map. As $\operatorname{GL}(V)=\mathbb{C}^{*}$ acts trivially on $\operatorname{End}(V)$, and on $W$ by weight -1 , we have

$$
M_{\mathbb{P}^{2}}(r, 0,-1) \cong \operatorname{Tot}_{\mathbb{P}^{(r-1)}}\left(\mathcal{O}^{\oplus 2} \oplus \Omega\right) .
$$

Next we determine the variety $M^{1}(r, 0,-1)$. Recall that we have an isomorphism (cf. [21, Proposition 7.4])

$$
\phi: M^{0}(r, 0,-1) \stackrel{\cong}{\rightarrow} M_{\mathbb{P}^{2}}(r, 0,-1), \quad\left(B_{1}, B_{2}, d, i, j\right) \mapsto\left(d B_{1}, d B_{2}, d i, j\right) .
$$

The locus blown-up by $\xi_{0}^{+}$is given by

$$
M^{0}(r, 0,-1)_{1}=\left\{E \in M^{0}(r, 0,-1): \operatorname{hom}\left(\mathcal{O}_{C}(-1), E\right)=1\right\} .
$$


As the sheaf $\mathcal{O}_{C}(-1)$ corresponds to the representation (5.2) with $m=0$, we see that the locus (5.5) coincides with the subvariety

$$
\mathbb{P}^{(r-1)} \cong L:=\left(B_{1}=B_{2}=j=0\right) \subset M_{\mathbb{P}^{2}}(r, 0,-1)
$$

under the isomorphism (5.4), which is nothing but the zero section.

\subsection{Hilbert scheme of points}

In this subsection, we consider the moduli spaces with Chern character $(1,0,-n)$. In this case, the diagram $(1.1)$ connects the Hilbert schemes of points Hilb ${ }^{n}\left(\mathbb{C}^{2}\right)$ and $\operatorname{Hilb}^{n}\left(\hat{\mathbb{C}}^{2}\right)$. We first analyze the stability of ideal sheaves $I_{Z} \in \operatorname{Hilb}^{n}\left(\hat{\mathbb{C}}^{2}\right)$ (it is an ideal sheaf of a length $n$ subscheme $Z \subset \hat{\mathbb{P}}^{2}$ with $Z \cap l_{\infty}=\emptyset$ ).

Lemma 5.3 Let us take a point $I_{Z} \in \operatorname{Hilb}^{n}\left(\hat{\mathbb{C}}^{2}\right)$ and let $k$ be a length of $Z \cap C$. Then $I_{Z}$ is $k$-stable but not $(k-1)$-stable. Furthermore, its destabilizing sequence for $(k-1)$-stability is given as

$$
0 \rightarrow I_{W}(-C) \rightarrow I_{Z} \rightarrow \mathcal{O}_{C}(-k) \rightarrow 0
$$

for some length $(n-k)$ zero dimensional subscheme $W \subset \hat{\mathbb{P}}^{2}$ with $W \cap C=\emptyset$.

Proof The first statement follows from the proof of [17, Lemma 6.1]. For the second statement, let $\mathcal{O}_{W}$ be the kernel of the surjection $\mathcal{O}_{Z} \rightarrow \mathcal{O}_{Z \cap C}$. Then we have the following diagram.

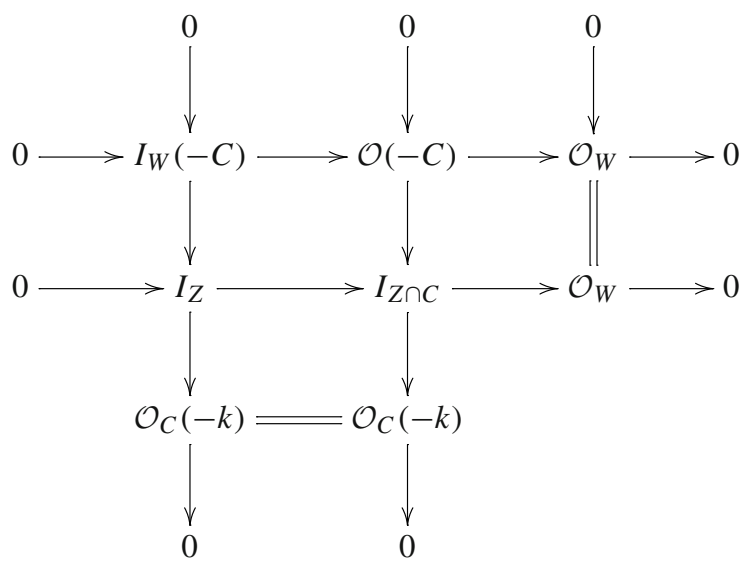

Furthermore, the sheaf $I_{W}(-C)$ is 0 -stable since $W \cap C=\emptyset$. Hence the second assertion follows.

5.2.1. When $n=2$, we have the following diagram:

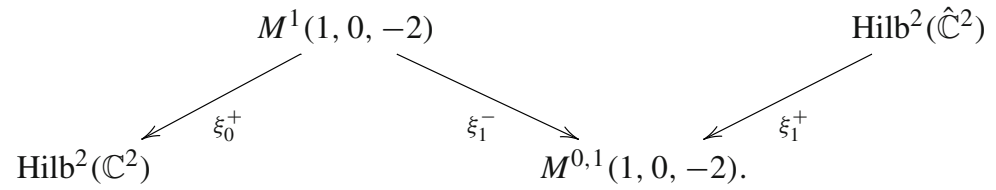

The properties of the diagram are summarized as follows (cf. [17, Theorem 1.4]): 
- $\xi_{1}^{+}$contracts $\operatorname{Hilb}^{2}(C) \cong \mathbb{P}^{2}$, and $\xi_{1}^{-}$contracts $\mathbb{P}^{1}$.

- The birational map $\operatorname{Hilb}^{2}\left(\hat{\mathbb{C}}^{2}\right) \rightarrow M^{1}(1,0,-2)$ is a standard flip.

- $\xi_{0}^{+}$is the blow-up at the codimension two subvariety

$$
\left\{I_{Y} \in \operatorname{Hilb}^{2}\left(\mathbb{C}^{2}\right): Y \ni 0\right\} \cong \hat{\mathbb{C}}^{2} .
$$

The derived category $D^{b}\left(\operatorname{Hilb}\left(\hat{\mathbb{C}}^{2}\right)\right)$ has the following semi-orthogonal decomposition:

$$
\begin{aligned}
D^{b}\left(\operatorname{Hilb}^{2}\left(\hat{\mathbb{C}}^{2}\right)\right) & =\left\langle D^{b}(\mathrm{pt}), D^{b}\left(M^{1}(1,0,-2)\right\rangle\right. \\
& =\left\langle D^{b}(\mathrm{pt}), D^{b}\left(\hat{\mathbb{C}}^{2}\right), D^{b}\left(\operatorname{Hilb}^{2}\left(\mathbb{C}^{2}\right)\right)\right\rangle \\
& =\left\langle D^{b}(\mathrm{pt}), D^{b}(\mathrm{pt}), D^{b}\left(\mathbb{C}^{2}\right), D^{b}\left(\operatorname{Hilb}^{2}\left(\mathbb{C}^{2}\right)\right)\right\rangle .
\end{aligned}
$$

5.2.2. Next we consider the case when $n=3$. First let us analyze geometry of

$$
M^{2}(1,0,-3) \stackrel{\xi_{2}^{-}}{\longrightarrow} M^{2,3}(1,0,-3) \stackrel{\xi_{2}^{+}}{\longleftarrow} \operatorname{Hilb}^{3}\left(\hat{\mathbb{C}}^{2}\right) .
$$

By Lemma 5.3, we have $\operatorname{Exc}\left(\xi_{2}^{+}\right)=\operatorname{Hilb}^{3}(C) \cong \mathbb{P}^{3}$,

$$
\operatorname{Exc}\left(\xi_{2}^{-}\right) \cong \mathbb{P}\left(\operatorname{Ext}^{1}\left(\mathcal{O}(-C), \mathcal{O}_{C}(-3)\right)\right)=\mathbb{P}^{2},
$$

and the diagram (5.6) is a standard flip.

Next we analyze the geometry of the morphisms $\xi_{1}^{ \pm}$. We have just seen that

$$
M^{2}(1,0,-3)=\left(\operatorname{Hilb}^{3}\left(\hat{\mathbb{C}}^{2}\right) \backslash \operatorname{Hilb}^{3}(C)\right) \cup \mathbb{P} \operatorname{Ext}^{1}\left(\mathcal{O}(-C), \mathcal{O}_{C}(-3)\right) .
$$

Take a non-trivial extension

$$
0 \rightarrow \mathcal{O}_{C}(-3) \rightarrow E_{2} \rightarrow \mathcal{O}(-C) \rightarrow 0,
$$

which defines a 2 -stable sheaf $\left[E_{2}\right] \in M^{2}(1,0,-3)$. We claim that we have the equality $\operatorname{hom}\left(E_{2}, \mathcal{O}_{C}(-2)\right)=1$. Indeed, by applying the functor $\operatorname{Hom}\left(-, \mathcal{O}_{C}(-2)\right)$ to the exact sequence (5.7), we have the exact sequence

$$
0 \rightarrow \operatorname{Hom}\left(E_{2}, \mathcal{O}_{C}(-2)\right) \rightarrow \operatorname{Hom}\left(\mathcal{O}_{C}(-3), \mathcal{O}_{C}(-2)\right) \rightarrow \operatorname{Ext}^{1}\left(\mathcal{O}(-C), \mathcal{O}_{C}(-2)\right),
$$

which proves the claim. By a standard diagram chasing, we can see that $E_{2}$ fits into the exact sequence

$$
0 \rightarrow I_{p}(-C) \rightarrow E_{2} \rightarrow \mathcal{O}_{C}(-2) \rightarrow 0
$$

for some point $p \in C$. Combining with Lemma 5.3, we conclude that

$$
\begin{aligned}
& \operatorname{Exc}\left(\xi_{1}^{+}\right)=\bigcup_{p \in \hat{\mathbb{C}}^{2}} \mathbb{P}\left(\operatorname{Ext}^{1}\left(\mathcal{O}_{C}(-2), I_{p}(-C)\right)\right), \\
& \operatorname{Exc}\left(\xi_{1}^{-}\right)=\bigcup_{p \in \hat{\mathbb{C}}^{2}} \mathbb{P}\left(\operatorname{Ext}^{1}\left(I_{p}(-C), \mathcal{O}_{C}(-2)\right)\right),
\end{aligned}
$$

which are $\mathbb{P}^{2}$-bundle, $\mathbb{P}^{1}$-bundle over $\hat{\mathbb{C}}^{2}$, respectively. The diagram

$$
M^{1}(1,0,-3) \stackrel{\xi_{1}^{-}}{\longrightarrow} M^{1,2}(1,0,-3) \stackrel{\xi_{1}^{+}}{\longleftarrow} M^{2}(1,0,-3)
$$

is a family of standard flips parametrized by $\hat{\mathbb{C}}^{2}$. 
Finally, let us consider the morphism $\xi_{0}^{+}: M^{1}(1,0,-3) \rightarrow \operatorname{Hilb}^{3}\left(\mathbb{C}^{2}\right)$. There are two types of objects in $M^{1}(1,0,-3)$ :

(1) an ideal sheaf $I_{Z}$, where $Z \subset \hat{\mathbb{C}}^{2}$ is a length 3 zero dimensional subscheme with $Z \cap C=$ $\{\mathrm{pt}\}$.

(2) an object $E_{1} \in \mathbb{P}\left(\operatorname{Ext}^{1}\left(I_{p}(-C), \mathcal{O}_{C}(-2)\right)\right)$, where $p \in \hat{\mathbb{C}}^{2}$.

Let us consider a sheaf $E_{1}$ of type (2). By a computation similar as above, we can see that hom $\left(E_{1}, \mathcal{O}_{C}(-1)\right) \leq 2$. Again, by a simple diagram chasing, we have the following possibilities:

- When $\operatorname{hom}\left(E_{1}, \mathcal{O}_{C}(-1)\right)=1, E_{1}$ fits into a exact sequence

$$
0 \rightarrow I_{p, q}(-C) \rightarrow E_{1} \rightarrow \mathcal{O}_{C}(-1) \rightarrow 0
$$

for some $q \in C$ with $q \neq p$.

- When $\operatorname{hom}\left(E_{1}, \mathcal{O}_{C}(-1)\right)=2, E_{1}$ fits into an exact sequence

$$
0 \rightarrow \mathcal{O}(-2 C) \rightarrow E_{1} \rightarrow \mathcal{O}_{C}(-1)^{\oplus 2} \rightarrow 0 .
$$

As a summary, we list up the properties of the morphism $\xi_{0}^{+}: M^{1}(1,0,-3) \rightarrow \operatorname{Hilb}^{3}\left(\mathbb{C}^{2}\right)$ :

- We have $\xi_{0}^{+}\left(\operatorname{Exc}\left(\xi_{0}^{+}\right)\right)=M^{1}(1,0,-2) \cap M^{2}(1,0,-2)=M^{1,2}(1,0,-2)$, which has a single singular point $o \in M^{1,2}(1,0,-2)$.

- For a point $p \in M^{1,2}(1,0,-2)$, the fiber of $\xi_{0}^{+}$is given as

$$
\left(\xi_{0}^{+}\right)^{-1}(p) \cong \begin{cases}F_{o}:=\mathbb{P}^{2} & (p=o) \\ F_{p}:=\mathbb{P}^{1} & (p \neq o) .\end{cases}
$$

- The normal bundles of fibers of $\xi_{0}^{+}$are given as

$$
\begin{aligned}
& \mathcal{N}_{F_{o} / M^{1}(1,0,-3)} \cong \Omega_{\mathbb{P}^{2}}(-1)^{\oplus 2}, \\
& \mathcal{N}_{F_{p} / M^{1}(1,0,-3)} \cong \mathcal{O}_{\mathbb{P}^{1}}(-1) \oplus \mathcal{O}_{\mathbb{P}^{1}}^{\oplus 4} \quad(p \neq o) .
\end{aligned}
$$

- The moduli space $M^{1}(1,0,-3)$ is isomorphic to $\mathrm{Bl}_{M^{1,2}(1,0,-2)} \operatorname{Hilb}^{3}\left(\mathbb{C}^{2}\right)$.

Using the semi-orthogonal decompositions for standard flips and blow-ups of codimension two Cohen-Macaulay subschemes (cf. [14, 3.1.2]), we have

$$
\begin{aligned}
& D^{b}\left(\operatorname{Hilb}^{3}\left(\hat{\mathbb{C}}^{2}\right)\right) \\
& \quad=\left\langle D^{b}(\mathrm{pt}), D^{b}(\mathrm{pt}), D^{b}(\mathrm{pt}), D^{b}\left(\mathbb{C}^{2}\right), D^{b}\left(\mathbb{C}^{2}\right), D^{b}\left(\operatorname{Hilb}^{2}\left(\mathbb{C}^{2}\right)\right), D^{b}\left(\operatorname{Hilb}^{3}\left(\mathbb{C}^{2}\right)\right)\right\rangle .
\end{aligned}
$$

5.2.3. We give the first example where the Grassmannian variety (which is not the projective space) appears as a fiber: let us consider a non-trivial extension

$$
0 \rightarrow \mathcal{O}(-2 C) \rightarrow E \rightarrow \mathcal{O}_{C}(-2)^{\oplus 2} \rightarrow 0 .
$$

The sheaf $E$ is 2-stable but not 1-stable, and has a Chern character $(1,0,-5)$. Noting that $\operatorname{ext}^{1}\left(\mathcal{O}_{C}(-2), \mathcal{O}(-2 C)\right)=4$, we have $\operatorname{Gr}(2,4) \subset M^{2}(1,0,-5)$ and it is contracted by the morphism $\xi_{1}^{+}: M^{2}(1,0,-5) \rightarrow M^{1,2}(1,0,-5)$.

Acknowledgements I would like to thank Professors Jim Bryan and Yukinobu Toda for fruitful discussions. This work was supported by Grant-in-Aid for JSPS Research Fellow 17J00664. Finally, I would like to thank the referee for various suggestions and comments to the previous version of this paper. 
Open Access This article is licensed under a Creative Commons Attribution 4.0 International License, which permits use, sharing, adaptation, distribution and reproduction in any medium or format, as long as you give appropriate credit to the original author(s) and the source, provide a link to the Creative Commons licence, and indicate if changes were made. The images or other third party material in this article are included in the article's Creative Commons licence, unless indicated otherwise in a credit line to the material. If material is not included in the article's Creative Commons licence and your intended use is not permitted by statutory regulation or exceeds the permitted use, you will need to obtain permission directly from the copyright holder. To view a copy of this licence, visit http://creativecommons.org/licenses/by/4.0/.

\section{References}

1. Andreatta, M., Wiśniewski, J.A.: A view on contractions of higher-dimensional varieties. Algebraic Geometry-Santa Cruz 1995, Volume 62 of Proceedings of Symposia in Pure Mathematics, pp. 153-183. American Mathematical Society, Providence (1997)

2. Anno, R., Logvinenko, T.: On adjunctions for Fourier-Mukai transforms. Adv. Math. 231(3-4), 20692115 (2012)

3. Arbarello, E., Cornalba, M., Griffiths, P.A., Harris, J.: Geometry of Algebraic Curves Vol. I, Volume 267 of Grundlehren der Mathematischen Wissenschaften [Fundamental Principles of Mathematical Sciences]. Springer, New York (1985)

4. Ballard, M.R.: Wall crossing for derived categories of moduli spaces of sheaves on rational surfaces. Algebr. Geom. 4(3), 263-280 (2017)

5. Bondal, A., Orlov, D.: Semiorthogonal decomposition for algebraic varieties. alg-geom/9506012 (arXiv e-prints) (1995)

6. Bridgeland, T.: Flops and derived categories. Invent. Math. 147(3), 613-632 (2002)

7. Bruzzo, U., Markushevish, D.: Moduli of framed sheaves on projective surfaces. Doc. Math. 16, 399-410 (2011)

8. Buchweitz, R.-O., Leuschke, G.J., Van den Bergh, M.: Non-commutative desingularization of determinantal varieties, II: arbitrary minors. Int. Math. Res. Not. 9, 2748-2812 (2016)

9. Donovan, W., Segal, E.: Window shifts, flop equivalences and Grassmannian twists. Compos. Math. 150(6), 942-978 (2014)

10. Ellingsrud, G., Göttsche, L.: Variation of moduli spaces and Donaldson invariants under change of polarization. J. Reine Angew. Math. 467, 1-49 (1995)

11. Friedman, R., Qin, Z.: Flips of moduli spaces and transition formulas for Donaldson polynomial invariants of rational surfaces. Commun. Anal. Geom. 3(1-2), 11-83 (1995)

12. Ginzburg, V.: Lectures on Nakajima's Quiver varieties. arXiv:0905.0686 (arXiv e-prints) (2009)

13. Huybrechts, D.: Fourier-Mukai Transforms in Algebraic Geometry. Oxford Mathematical Monographs. Oxford University Press, Oxford (2006)

14. Jiang, Q., Leung, N.C.: Derived category of projectivization and flops. arXiv:1811.12525 (arXiv e-prints) (2018)

15. Kawamata, Y.: $D$-equivalence and $K$-equivalence. J. Differ. Geom. 61(1), 147-171 (2002)

16. Kollár, J., Mori, S.: Birational Geometry of Algebraic Varieties, Volume 134 of Cambridge Tracts in Mathematics. Cambridge University Press, Cambridge (1998). (With the collaboration of C. H. Clemens and A. Corti, Translated from the 1998 Japanese original)

17. Koseki, N.: Perverse coherent sheaves on blow-ups at codimension 2 loci. Int. Math. Res. Not. (2019). https://doi.org/10.1093/imrn/rnz175

18. Koseki, N., Toda, Y.: Derived categories of Thaddeus pair moduli spaces via d-critical flips. arXiv:1904.04949 (arXiv e-prints) (2019)

19. Nakajima, H.: Instantons on ALE spaces, quiver varieties, and Kac-Moody algebras. Duke Math. J. 76(2), 365-416 (1994)

20. Nakajima, H.: Lectures on Hilbert Schemes of Points on Surfaces. University Lecture Series, vol. 18. American Mathematical Society, Providence (1999)

21. Nakajima, H., Yoshioka, K.: Perverse coherent sheaves on blow-up. I. A quiver description. Exploring New Structures and Natural Constructions in Mathematical Physics, vXolume 61 of Advanced Studies in Pure Mathematics, pp. 349-386. Mathematical Society of Japan, Tokyo (2011)

22. Nakajima, H., Yoshioka, K.: Perverse coherent sheaves on blow-up. II. Wall-crossing and Betti numbers formula. J. Algebr. Geom. 20(1), 47-100 (2011)

23. Nakajima, H., Yoshioka, K.: Perverse coherent sheaves on blowup, III: Blow-up formula from wallcrossing. Kyoto J. Math. 51(2), 263-335 (2011) 
24. Toda, Y.: Birational geometry for d-critical loci and wall-crossing in Calabi-Yau 3-folds. arXiv: 1805.00182 (arXiv e-prints) (2018)

25. Toda, Y.: Semiorthogonal decompositions of stable pair moduli spaces via d-critical flips. arXiv:1805.00183 (arXiv e-prints) (2018)

26. Weyman, J.: Cohomology of Vector Bundles and Syzygies. Cambridge Tracts in Mathematics, vol. 149. Cambridge University Press, Cambridge (2003)

Publisher's Note Springer Nature remains neutral with regard to jurisdictional claims in published maps and institutional affiliations. 